\title{
Estudio de los microvertebrados del yacimiento de Praileaitz I (Deba, Gipuzkoa)
}

\author{
Praileaitz I (Deba, Gipuzkoa) aztarnategiaren mikroornodunen ikerketa \\ Study of small vertebrates from Praileaitz I (Deba, Gipuzkoa)
}

PALABRAS CLAVE: Microvertebrados, Pleistoceno, Holoceno, Praileaitz I, Cornisa Cantábrica.

GAKO-HITZAK: Mikrornodunak, Pleistozenoa, Holozenoa, Praileaitz I, Kantauriar Erlaitza.

KEYWORDS: Small vertebrates, Pleistocene, Holocene, Praileaitz I, Cantabrian Range.

\section{Naroa GARCIA-IBAIBARRIAGA ${ }^{(1)}$, Salvador BAILON(2), Juan ROFES(3), Amaia ORDIALES(4), Aitziber SUÁREZ-BILBAO(4), Xabier MURELAGA ${ }^{(4)}$}

\section{RESUMEN}

En este trabajo se presentan los resultados del estudio de los restos de microvertebrados hallados en las excavaciones realizadas en el yacimiento de Praileaitz I (Deba, Gipuzkoa). Aun cuando en estas campañas no se recogieron muestras específicas para el estudio de los microvertebrados, hemos podido utilizar los residuos del estudio sedimentológico, abarcando un secuencia cronocultural que va desde el Solutrense al Epipaleolítico. De esta manera, se constata una asociación de microvertebrados formada por un mínimo de 13 taxones diferentes de micromamíferos (Microtus (Microtus) arvalis, Microtus (Microtus) agrestis, Microtus (Terricola) sp., Microtus (Alexandromys) oeconomus, Chionomys nivalis, Arvicola amphibius, Arvicola cf. sapidus, Clethrionomys glareolus, Apodemus sylvaticus-flavicollis, Glis glis, Talpa sp., Crocidura russula, Sorex minutus y Sorex araneus-coronatus), tres anfibios (Alytes cf. obstetricans, Bufo bufo y Rana temporariaiberica) y un reptil (cf. Lacerta). Las afinidades autoecológicas de la asociación de microvertebrados descrita indicarían unas condiciones frías durante el Magdaleniense, mientras que la situación sería más benigna durante el Epipaleolítico.

\section{LABURPENA}

Lan honetan Praileaitz I (Deba, Gipuzkoa) aztarnategian burututako indusketetan jasotako mikroornodunen azterketaren emaitzak azaltzen dira. Nahiz eta kanpaina hauetan mikroornodunen ikerketarako lagin zehatzik jaso ez zen arren, azterketa sedimentologikoen hondarrak erabili ahal izan ditugu. Honela, 13 mikrougaztun taxoi desberdinez (Microtus (Microtus) arvalis, Microtus (Microtus) agrestis, Microtus (Terricola) sp., Microtus (Alexandromys) oeconomus, Chionomys nivalis, Arvicola amphibius, Arvicola cf. sapidus, Clethrionomys glareolus, Apodemus sylvaticus-flavicollis, Glis glis, Talpa sp., Crocidura russula, Sorex minutus eta Sorex araneus-coronatus), hiru anfibioz (Alytes cf. obstetricans, Bufo bufo eta Rana temporaria-iberica) eta narrasti bakarraz (cf. Lacerta) osatutako mikroornodun multzoa definitu da. Aztertutako laginak Solutre Alditik Epipaleolitora doan tarte krono-kulturalari dagozkio. Afinitate autoekologikoen arabera esan dezakegu Magdalen Aldirako deskribatutako mikroornodunak baldintza hotzagoen adierazle direla. Epipaleolitokoak, berriz, baldintza klimatiko epelagoen seinale dira.

\section{ABSTRACT}

In this paper the small vertebrate assemblage from Praileaitz I (Deba, Gipuzkoa) is described. Although during the archaeological works were not taken specific samples for the microvertebrates study, we could use the residue from the sedimentological study, encompassing a chrono-cultural sequence from the Solutrean to the Mesolithic. A total of 13 different small mammal taxa have been successfully identified (Microtus (Microtus) arvalis, Microtus (Microtus) agrestis, Microtus (Terricola) sp., Microtus (Alexandromys) oeconomus, Chionomys nivalis, Arvicola amphibius, Arvicola cf. sapidus, Clethrionomys glareolus, Apodemus sylvaticus-flavicollis, Glis glis, Talpa sp., Crocidura russula, Sorex minutus and Sorex araneus-coronatus), appart from three amphibians (Alytes cf. obstetricans, Bufo bufo and Rana temporariaiberica) and one reptil (cf. Lacerta). The environmental conditions estimated on the basis of this microvertebrate association autoecological affinities suggested that during the Magdalenian period the climatic conditions would be colder than the one occurring in the area at the present day, while the climate became warmer during the Epipaleolithic.

\footnotetext{
(1) Departamento de Geografía, Prehistoria y Arqueología, Facultad de Letras, Euskal Herriko Unibertsitatea UPV/EHU, c/ Tomás y Valiente s/n, 01006 Vitoria-Gasteiz; naroa.garcia@ehu.es (2) UMR 7209 - 7194 (CNRS, MNHN) ; Muséum national d'Histoire naturelle, Sorbonne Universités, 55 rue Buffon, CP 56, 75005 Paris. (3) Archéozoologie, Archéobotanique: Sociétés, pratiques et environnements (UMR 7209), Sorbonne Universités, Muséum national d'Histoire naturelle, CNRS, CP56, 55 rue Buffon, 75005 Paris.

(4) Departamento de Estratigrafía y Paleontología, Facultad de Ciencia y Tecnología, Euskal Herriko Unibertsitatea UPV/EHU, Barrio Sarriena $\mathrm{s} / \mathrm{n}, 48940$ Leioa.
} 


\section{1.- INTRODUCCIÓN}

En la mayoría de los yacimientos del Cuaternario (exceptuando trampas naturales) de la Cornisa Cantábrica, los restos de macromamíferos acumulados están asociados a la actividad humana o a la de grandes carnívoros, y por lo tanto, su presencia relativa estará en muchas ocasiones condicionada por las apetencias cinegéticas y depredadoras de estos. Por su parte, los microvertebrados suelen ser cazados por mamíferos de pequeño tamaño, aves diurnas y aves nocturnas (ANDREWS, 1990). De estos tres grupos, las aves nocturnas son las que menos daño causan a los restos, por lo que este agente biológico suele ser generalmente el principal autor de las tafocenosis de los microvertebrados que se encuentran en el registro fósil. Al ser estas especies de apetencias bastante generalistas, la representatividad de los microvertebrados fósiles encontrados en los diferentes niveles se acerca al existente en el entorno de la cueva en el momento de la formación del deposito estudiado, por lo que el conjunto nos puede dar una idea de los cambios ambientales acaecidos a lo largo del registro estudiado, aun cuando solo se haya recuperado microfauna de algunos de los cuadros excavados.

Con el presente trabajo, se espera reconstruir la biodiversidad de microvertebrados en cada uno de los lechos, y estimar, a partir de las variaciones de la misma a lo largo del tiempo, los cambios ambientales que se dieron durante el periodo registrado en la serie estratigráfica de Praileaitz I.

\section{2.- METODOLOGÍA}

Aunque durante las campañas de excavación realizadas en el yacimiento de Praileaitz I no se recogieron muestras especificas para el estudio de los microvertebrados, hemos podido utilizar los residuos de tamaño de partícula superior a 0,5 mm provenientes del estudio sedimentológico. Estas muestras corresponderían a los cuadros $6 \mathrm{~A}$ y $14 \mathrm{E}$ del vestíbulo y $8 \mathrm{~F}^{\prime}$ y $10 \mathrm{~F}$ ' de la primera sala interior (Fig. 1). Además, contamos con muestras obtenidas durante la excavación propiamente dicha con el tamiz de $2 \mathrm{~mm}$.

Fig. 1. Planimetría del yacimiento de Praileaitz | (Deba, Gipuzkoa). En gris la ubicación de los cuadros muestreados para el estudio sedimentológico. / General plan of Praileaitz I site (Deba, Gipuzkoa). In grey the location of the squares sampled to the sedimentological study.
Las muestras del tamiz de $2 \mathrm{~mm}$, con las cuales se ha estimado la distribución de los restos en toda la superficie excavada, provienen de las tres zonas excavadas (vestíbulo, primera sala interior y segunda sala interior). Debido a la cantidad de restos de microvertebrados recuperados, se ha calculado el peso de los mismos con una báscula marca Salter modelo EW-300B, con una precisión de 0,1 g, a fin de estimar su riqueza en los diferentes cuadros excavados. Para realizar las figuras de las isolíneas de número de restos por cuadro, se han utilizado los puntos medios de cada cuadro para obtener las coordenadas $\mathrm{X}$ e $\mathrm{Y}$, mientras que la $\mathrm{Z}$ proviene del peso de los restos de microvertebrados. El peso total de las 857 bolsas con restos de microfauna procedentes del vestíbulo es de 1998,1 g; 1336,1 $\mathrm{g}$ el de las 130 bolsas de la primera sala interior y $91,13 \mathrm{~g}$ el de las 102 bolsas

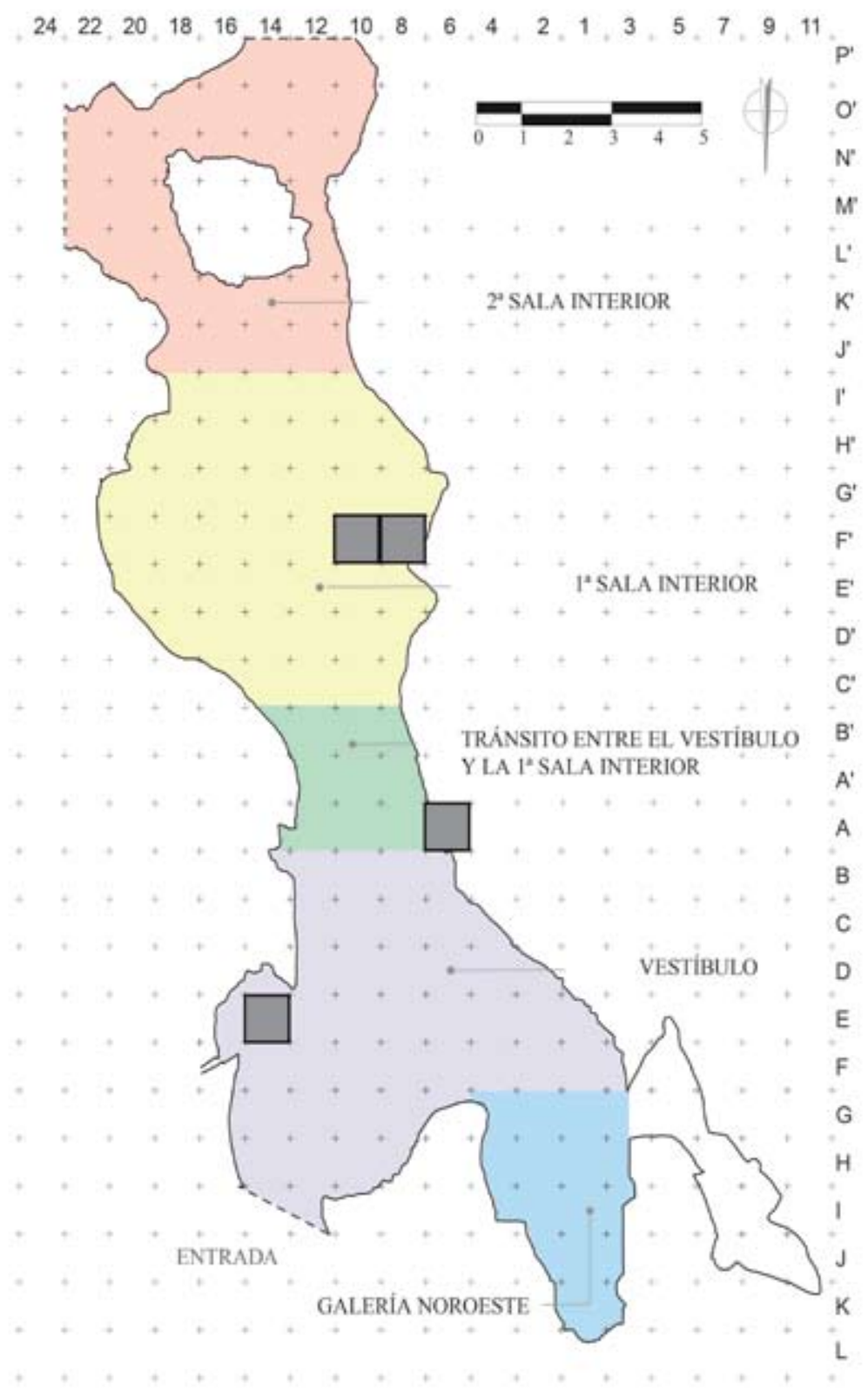


de la segunda sala interior. Estas muestras no han sido tenidas en cuenta a la hora de realizar las interpretaciones paleoecológicas, puesto que muchos de los restos inferiores a $2 \mathrm{~mm}$ se habrán perdido, por lo que es probable que no estén representadas todas las especies. De todas maneras, siendo el sesgo el mismo para todos los cuadros excavados, es posible utilizar los pesos para estimar si la distribución de los restos en el yacimiento se debe al azar o si sigue algún patrón.

Para realizar el estudio de la asociación de microvertebrados, y determinar las proporciones en las que se encuentran representadas las diferentes especies, se han utilizado las muestras recogidas para el estudio sedimentológico (Tabl. 1). De estas muestras nos hemos quedado con las fracciones de tamaño de grano superior a $0,5 \mathrm{~mm}$, triadas con la ayuda de una lupa binocular Nikon SMZ-U. Al tratarse de muestras para sedimentología, la cantidad de sedimento recogido ha sido similar en las 10 muestras estudiadas (alrededor de 5 kg). En conjunto, Praileaitz I es muy rico en restos de microvertebrados, contabilizándose 82573 restos identificables (Fig. 2). La muestra más rica correspondería al V6, con 59.680 restos recuperados, mientras que V4 sería la más pobre, con únicamente 67 restos.

\begin{tabular}{|c|c|c|c|c|c|}
\hline Sigla & Cuadro & Talla & Lecho unificado & Zona del yacimiento & Cronología cultural \\
\hline V1 & $6 \mathrm{~A}$ & 1 & 1 & Vestíbulo & Superficial \\
\hline V2 & $6 \mathrm{~A}$ & 3 & 3 & Vestíbulo & Epipalerficial \\
\hline V3 & $6 \mathrm{~A}$ & 5 & 5 & Vestíbulico \\
\hline V4 & $6 \mathrm{~A}$ & 7 & 7 & Vestíbulo & Epipaleolítico \\
\hline V5 & $6 \mathrm{~A}$ & 13 & 9 & Vestíbulo & Epipaleolítico \\
\hline V6 & $6 \mathrm{~A}$ & 17 & 11 & Vestíbulo & Magdaleniense inferior \\
\hline V7 & $14 \mathrm{E}$ & 20 & 11 & Vestíbulo & Magdaleniense inferior \\
\hline S1 & $8 F^{\prime}$ & 7 & 7 & $1^{\circ}$ Sala interior & Magdaleniense inferior \\
\hline S2 & $8 F^{\prime}$ & 7 & 7 & $1^{\circ}$ Sala interior & Magdaleniense inferior \\
\hline S3 & $10 F^{\prime}$ & 9 & 13 & $1^{\circ}$ Sala interior & Solutrense \\
\hline
\end{tabular}

Tabl. 1. Relación de las siglas utilizadas para cada muestra del yacimiento de Praileaitz I (Deba, Gipuzkoa), su situación estratigráfica y atribución crono-cultural. / Relation of the acronyms used for each sample from Praileaitz I site (Deba, Gipuzkoa), their stratigraphic location and chrono-cultural attribution.

Los restos han sido identificados siguiendo los criterios generales en micropaleontología. La atribución específica del material de reptiles y anfibios se basa principalmente en los elementos diagnósticos: vértebra troncal en el reptil y húmero, ilion, escápula y vértebras en el caso de los anuros. Para los mamíferos se ha utili-

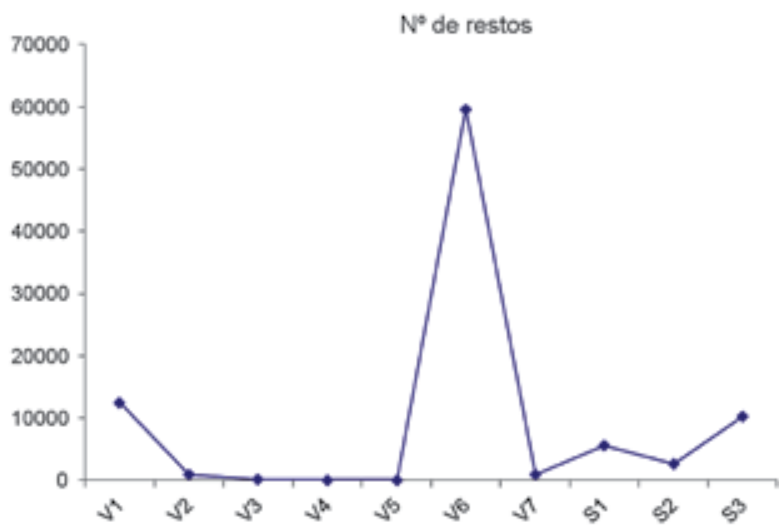

Fig. 2. Número de restos de microvertebrados de las muestras del yacimiento de Praileaitz I (Deba, Gipuzkoa). / Number of small vertebrates remains from Praileaitz I site (Deba, Gipuzkoa). zado la morfología y talla de la dentición. La clasificación taxonómica de los anfibios y reptiles sigue el trabajo de Carretero et al. (2011) y los criterios de determinación osteológica a Bailon (1991 y 1999) y Blain (2009). Por su parte, la nominación sistemática de los mamíferos se basa en Wilson y Reeder (2005) y Tesakov et al. (2010). Para la nomenclatura utilizada en la descripción de los dientes de arvicólidos se han utilizado los trabajos de Van der Meulen (1973) y Heinrich (1978), el de Reumer (1984) para los insectívoros y el de Daams (1981) para los glíridos. Los fósiles han sido agrupados usando el método del número mínimo de individuos (NMI), contabilizando el elemento más diagnóstico (Tabl. 2).

Basándonos en el índice SDQ propuesto por Heinrich (1982), es posible diferenciar entre las especies del género Arvicola. Por su parte, Pedro Castaños (comunicación personal) identificó 36 restos de marmota, sobre todo fragmentos de incisivos, entre los restos de la macrofauna. No se hace descripción de los mismos en este trabajo al haber sido incluidos en los contajes del capítulo correspondiente a los macrovertebrados de esta misma monografía.

Los requisitos ambientales de cada uno de los taxones de micromamíferos descritos en el yacimiento de Praileaitz I provienen de los trabajos de Cuenca-Bescós et al. (2008), Pemán (1985), Pokines (1998), Rofes et al. 


\begin{tabular}{|l|c|c|c|c|c|c|c|c|c|c|}
\hline NMI & S1 & S2 & S3 & V1 & V2 & V3 & V4 & V5 & V6 & V7 \\
\hline Microtus (Microtus) arvalis & 14 & 59 & 19 & 1 & 0 & 0 & 0 & 0 & 161 & 1 \\
\hline Microtus (Microtus) agrestis & 2 & 6 & 0 & 0 & 0 & 0 & 0 & 0 & 21 & 0 \\
\hline Microtus (Terricola) sp. & 17 & 18 & 10 & 1 & 0 & 0 & 0 & 0 & 27 & 1 \\
\hline Microtus (Alexandromys) oeconomus & 27 & 30 & 12 & 1 & 0 & 0 & 0 & 0 & 18 & 3 \\
\hline Chionomys nivalis & 2 & 3 & 0 & 0 & 0 & 0 & 0 & 0 & 6 & 0 \\
\hline Arvicola amphibius & 1 & 4 & 1 & 0 & 0 & 0 & 0 & 0 & 0 & 0 \\
\hline Arvicola cf. sapidus & 0 & 0 & 0 & 1 & 0 & 0 & 0 & 0 & 0 & 0 \\
\hline Clethrionomys glareolus & 0 & 0 & 0 & 1 & 0 & 0 & 0 & 0 & 0 & 0 \\
\hline Apodemus sylvaticus-flavicollis & 1 & 3 & 1 & 9 & 3 & 1 & 1 & 1 & 0 & 0 \\
\hline Glis glis & 0 & 0 & 0 & 1 & 0 & 0 & 0 & 0 & 0 & 0 \\
\hline Talpa sp. & 0 & 0 & 0 & 8 & 0 & 1 & 0 & 0 & 0 & 0 \\
\hline Crocidura russula & 2 & 4 & 1 & 0 & 0 & 0 & 0 & 0 & 0 & 0 \\
\hline Sorex minutus & 2 & 5 & 1 & 1 & 0 & 0 & 0 & 0 & 5 & 1 \\
\hline Sorex araneus-coronatus & 20 & 45 & 22 & 6 & 1 & 1 & 0 & 0 & 41 & 4 \\
\hline Alytes cf. obstetricans & & & 1 & & & & & & \\
\hline Rana temporaria-iberica & 3 & 1 & 3 & & & & & & 3 & \\
\hline Bufo bufo & & 1 & 2 & & & & & & \\
\hline cf. Lacerta & 91 & 179 & 73 & 31 & 4 & 3 & 1 & 1 & 282 & 11 \\
\hline TOTAL de NMI & & & 1 & & & \\
\hline
\end{tabular}

Tabl. 2. Número mínimo de individuos estimados en cada una de las muestras estudiadas en el yacimiento de Praileaitz I (Deba, Gipuzkoa). / Minimum number of individuals estimated in each of the samples studied from Praileaitz I site (Deba, Gipuzkoa).

(2013) y Sesé (2005). Por su parte, la asignación de la distribución y datos ambientales de la herpetofauna sigue los trabajos de García-París et al. (2004), Pleguezuelos et al. (2004) y Salvador (1998). Las estimaciones de las variaciones de paleotemperatura, se han basado en la proporción de las especies afines a ambientes más fríos con respecto a las de condiciones más cálidas (Tabl. 3).

\section{3.- TAFONOMÍA}

A fin de establecer la formación del conjunto de restos de anfibios y reptiles de Praileaitz I, el estudio de las trazas de digestión y fragmentación sigue los criterios de Pinto-Llona y Andrews (1999). Se han observado trazas de digestión en algunos huesos como el urostilo, ilion o húmero de los anuros, aunque su intensidad es moderada. En cuanto a la fracturación, es moderadamente significativa en el caso de los huesos largos. Al igual que sucede con los anfibios, en el caso del único resto de reptil encontrado podemos resaltar que éste muestra ligeras trazas de digestión. Por lo tanto, a partir de estos dos parámetros, probablemente una parte de los materiales de anfibios estudiados correspondería a individuos muertos in situ, mientras que algunos otros restos de anfibios, y el único resto de lacértido, presentan huellas que bien podrían corresponder a las de un depredador como Tyto alba (categoría 1 sensu Andrews, 1990).

En el caso de los micromamíferos, si se observan las proporciones relativas en las que se han preservado los

\begin{tabular}{|l|c|c|c|}
\hline & Frío & Cálido & Euritermo \\
\hline Microtus (Microtus) arvalis & & & $\mathrm{x}$ \\
\hline Microtus (Microtus) agrestis & & & $\mathrm{x}$ \\
\hline Microtus (Terricola) sp. & $\mathrm{x}$ & & \\
\hline $\begin{array}{l}\text { Microtus (Alexandromys) } \\
\text { oeconomus }\end{array}$ & $\mathrm{x}$ & & \\
\hline Chionomys nivalis & $\mathrm{x}$ & & \\
\hline Arvicola amphibius & & & $\mathrm{x}$ \\
\hline Arvicola cf. sapidus & & & $\mathrm{x}$ \\
\hline Clethrionomys glareolus & & $\mathrm{x}$ & \\
\hline $\begin{array}{l}\text { Apodemus sylvaticus- } \\
\text { flavicollis }\end{array}$ & & $\mathrm{x}$ & \\
\hline Glis glis & & $\mathrm{x}$ & \\
\hline Talpa sp. & & $\mathrm{x}$ & $\mathrm{x}$ \\
\hline Crocidura russula & $\mathrm{x}$ & & \\
\hline Sorex minutus & $\mathrm{x}$ & & \\
\hline Sorex araneus-coronatus & & \\
\hline
\end{tabular}

Tabl. 3. Estimaciones de las afinidades ecológicas utilizadas para cada uno de los taxones de micromamíferos presentes en el yacimiento de Praileaitz I (Deba, Gipuzkoa). / Estimations of the ecological affinities used for each of the small mammal taxa from Praileaitz I site (Deba, Gipuzkoa).

diferentes elementos anatómicos en Praileaitz I, es posible diferenciar dos grupos. En el primer grupo (muestras V3, V4 y V5) las muestras son más pobres y no están representados todos los elementos óseos (Fig. 3B). Por ello, la proporción relativa de los pocos restos que se han 

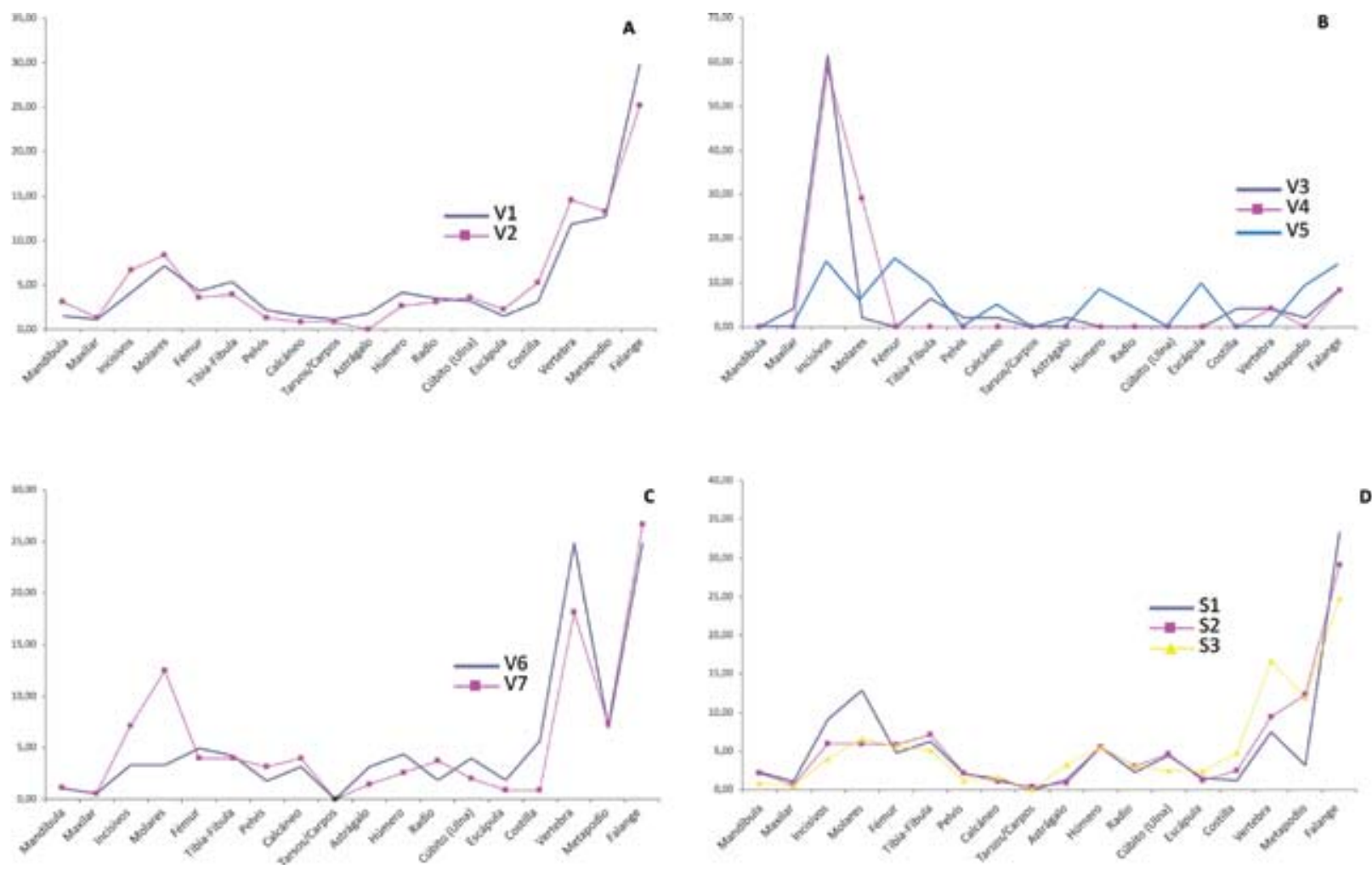

Fig. 3. $\mathrm{N}$ Proporción relativa de los diferentes elementos anatómicos de los microvertebrados del yacimiento de Praileaitz I (Deba, Gipuzkoa). / Relative proportions of the different anatomic elements of the microvertebrates from the Praileaitz I site (Deba, Gipuzkoa).

conservado en este grupo es mucho más alta que en el segundo (resto de las muestras), donde la mayoría de los elementos está presente en mayor o menor medida (Fig. 3A, 3C y 3D). Así, las curvas obtenidas de la representación relativa de los elementos anatómicos son distintas, lo cual indica que la historia tafonómica que han tenido estos dos grupos ha sido diferente. Con estas curvas es posible estimar el grado de alteración que han sufrido los restos antes de su enterramiento (ANDREWS, $1990 \mathrm{y}$ ANDREWS, EVANS, 1983). Aunque durante el procesado del material, i.e. extracción del sedimento, transporte y lavado-tamizado, también suelen desprenderse los molares de las mandíbulas y los maxilares, normalmente las diferencias considerables entre las proporciones de estos elementos anatómicos suelen indicar un transporte importante de los mismos. De esta manera, los lechos

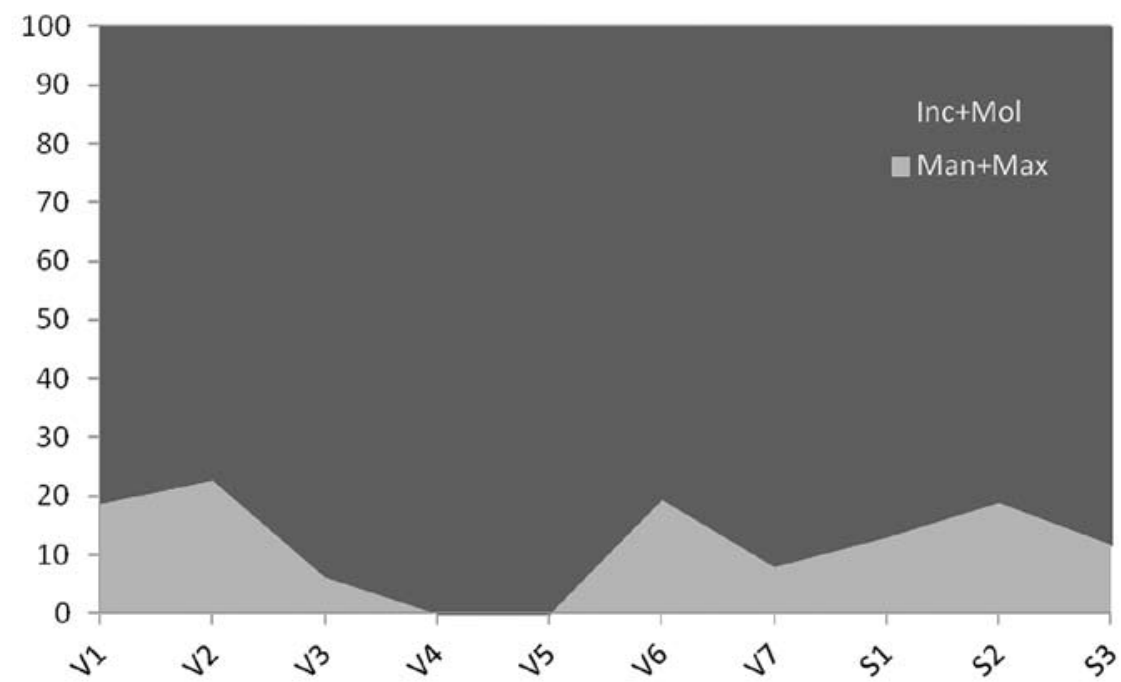

Fig. 4. Proporción relativa de la suma de incisivos y molares respecto a la de mandíbulas y maxilares de los micromamíferos del yacimiento de Praileaitz I (Deba, Gipuzkoa). / Relative proportion of the sum of incisors and molars regarding the jaws and maxillary of the small mammals from Praileaitz I site (Deba, Gipuzkoa). 
pertenecientes al primer grupo de muestras presentan una mayor proporción relativa de dientes sueltos que los del segundo grupo (Fig. 4).

La riqueza en número de restos concuerda con los datos anteriormente señalados, siendo las muestras del primer grupo (V3, V4 y V5) las más pobres del registro (Fig. 2). Estos datos son importantes para interpretar las muestras V3, V4 y V5, ya que, además de ser las más pobres, parece que han sufrido procesos tafonómicos diferentes a los del resto del yacimiento (Fig. 3) y un mayor transporte desde la zona de acumulación inicial generada por el agente biológico (Fig. 4). Hay que destacar que la muestra V6 presenta una riqueza notablemente superior a la del resto de las muestras estudiadas. Es posible interpretar este tipo de picos como equivalentes a momentos en los que no hay ocupación humana. Por ejemplo, tenemos una variación similar en el nivel con enterramiento del yacimiento de Peña Larga (Cripán, Álava) (ROFES et al., 2013).

Las acumulaciones puntuales o no aleatorias de restos de microvertebrados pueden generarse mediante diversos procesos, como por ejemplo las generadas por las aves rapaces o por las corrientes de agua en la zona de entrada de un sumidero. En estos casos, el transporte hacia las zonas interiores es generalmente realizado por pequeños flujos de agua o en menor medida por parte de otros organismos como los humanos u otros grandes mamíferos, generando, en este caso, una distribución aleatoria de los restos.

Las muestras obtenidas con la criba de más de 2 $\mathrm{mm}$ en la superficie de las tres zonas excavadas en Praileaitz I, permiten observar dos patrones principales según la riqueza de restos por cuadro. En algunos casos, la distribución de los restos en la superficie excavada no es aleatoria y se concentra en un cuadro concreto, mientras que en otros casos la acumulación se da en más de un cuadro a la vez. La zona del vestíbulo presenta una concentración no aleatoria, a excepción de los lechos 6, 20 y 22-24 (Anexo I). Por su parte, los lechos 2, 4-7 y 9 de la primera sala interior, resultan demasiado pobres para reflejar algún patrón. Aún así, las muestras de los lechos 10-13 son más ricas y en ellas se observa una repartición generalizada en diversos cuadros (Anexo II). Por último, la segunda sala interior presenta una distribución similar a la observada en los lechos más antiguos de la primera sala interior (Anexo III).

En resumen, la conservación relativa de los diferentes elementos anatómicos estimados en el vestíbulo, a excepción de las muestras V3, V4 y V5, nos indica que los restos no han sufrido un fuerte transporte (Figs. 3 y 4). La distribución de los restos en la superficie excavada se encuentra, en su mayoría, concentrada en ciertas zonas del vestíbulo, mientras que en las salas interiores está más repartida (Anexos I, II y III). La posición de las tres zonas excavadas cuando se generó el depósito debió ser similar a la actual, siendo el vestíbulo la zona de entrada a la cavidad y por lo tanto la más propicia para que se instalaran en ella los habituales agentes acumuladores de restos de microvertebrados, como las rapaces. Con todo, se plantea como hipótesis que los agentes biológicos podrían haber vivido en las repisas que rodean las paredes del vestíbulo, acumulándose los desechos de sus presas en la base de las mismas. Los restos habrían sido transportados a las salas interiores por algún agente tractivo como el agua. No es posible descartar que durante la formación de los lechos más antiguos de la primera sala interior entrasen depredadores como rapaces y que generasen algún acumulo de restos, aunque nos decantamos por la hipótesis de que éste se debe al transporte. La segunda sala interior es la más pobre en restos posiblemente por su lejanía respecto al vestíbulo, la zona principal de acumulación de restos.

\section{4.- SISTEMÁTICA}

Clase MAMMALIA LINNAEUS, 1758

Orden RODENTIA BOWDICH, 1821

Familia Cricetidae MurRAY, 1866

Subfamilia Arvicolinae Gray, 1821

Género Microtus SchRANK, 1798

Subgénero Microtus ScHRANK, 1798

Microtus (Microtus) arvalis (PALLAS 1778) (Fig. 5.1-2)

Esta especie se encuentra bien representada en todos los lechos, a excepción de los epipaleolíticos, en los cuales no se ha encontrado resto alguno. El M/1 presenta una disposición casi simétrica y en paralelo a los t4-t5 y t6-t7, mientras que los ángulos entrantes tienen una disposición similar a los salientes.

Al ser una especie más oportunista, el topillo campesino presenta una mayor repartición que su congénere (CUENCA-BESCÓS et al., 2008). Vive en numerosas zonas de Europa, y en la Península lbérica se encuentra en el Sistema Ibérico, Sistema Central, Meseta Norte y norte de la Península, pero nunca en la zona mediterránea. Normalmente habita en zonas abiertas con numerosa vegetación arbustiva y herbácea (SESÉ, 2005).

Microtus (Microtus) agrestis (LINNAEUS, 1761) (Fig. 5.3)

Al igual que el topillo campesino, esta especie está ausente en los lechos epipaleolíticos. A diferencia de éste, el M/1 tiene un complejo anterior con 4 triángulos casi cerrados, mientras que tanto los triángulos t4 y t5, como $t 6$ y 7 7, son asimétricos y alternantes. Por su parte, los ángulos entrantes presentan una fuerte alternancia.

El topillo agreste también tiene en la actualidad una amplia distribución en Europa, llegando más al norte que M. (M) arvalis. En la Península Ibérica es buen indicador del clima atlántico, ocupando unicamente la zona norte (ARRIZABALAGA et al., 1986). Habita una amplia diversidad de bosques, desde encinares hasta hayedos. Tambien puede vivir en zonas abiertas de poca vegetación, como praderas húmedas y juncales (SESÉ, 2005). 
1

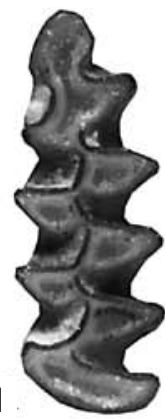

2
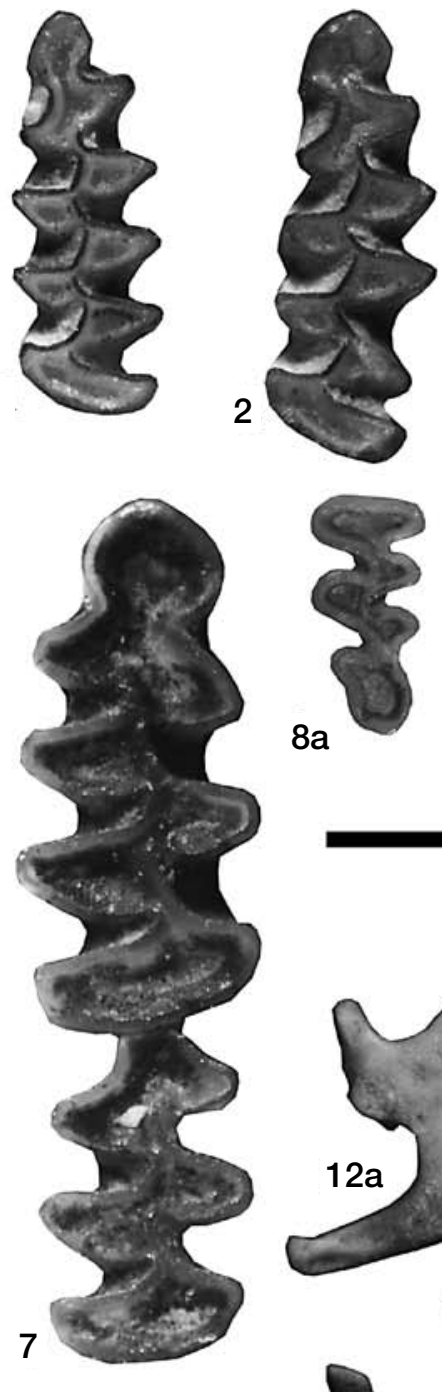

$8 b$

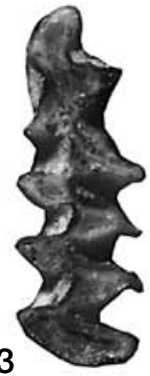

4
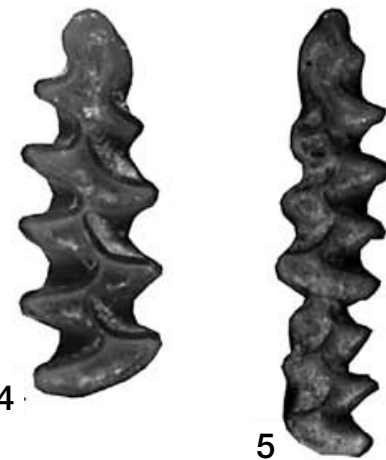

6

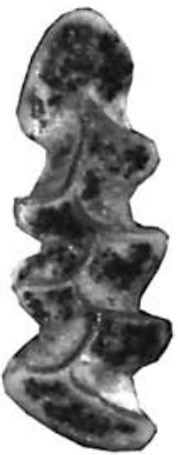

10

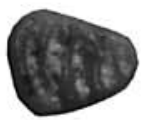

11

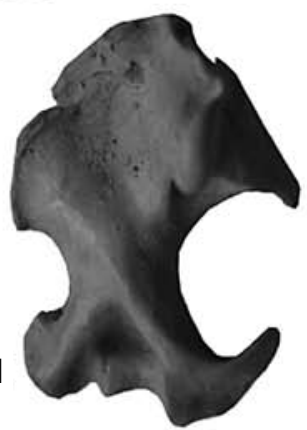

$12 b$
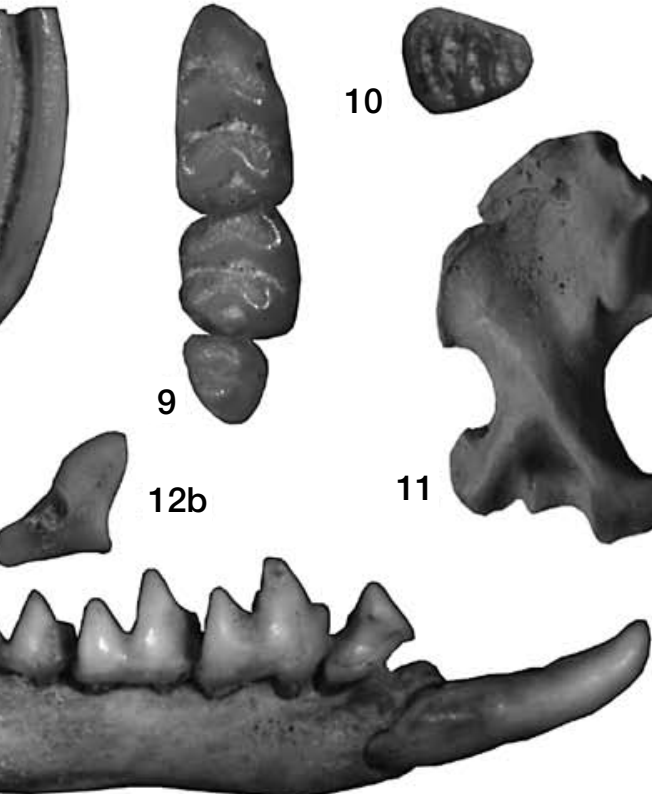

$12 \mathrm{a}$
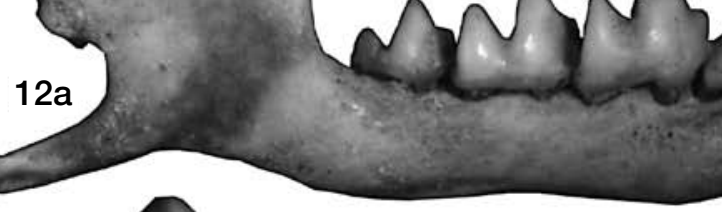

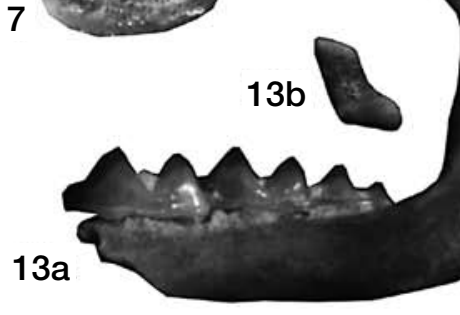

$14 \mathrm{a}$

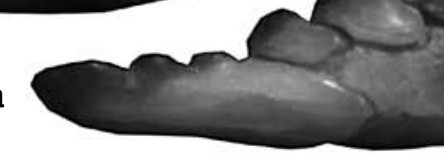

Fig. 5. Algunos de los restos de micromamíferos identificados en el yacimiento de Praileaitz I (Deba, Gipuzkoa). Microtus (Microtus) arvalis $1 \mathrm{M} / 1$ izquierdo $2 \mathrm{M} / 1$ izquierdo; Microtus (Microtus) agrestis $3 \mathrm{M} / 1$ izquierdo; Microtus (Terricola) sp. 4 M/1 derecho; Chionomys nivalis 5 M/1 izquierdo; Arvicola amphibius 6 M/1-2 derechos; Clethrionomys glareolus 7a M3/ derecho 7b ídem, vista lingual; Microtus (Alexandromys) oeconomus $\mathbf{8}$ M/1-2 izquierdos; Apodemus sylvaticus-flavicollis $\mathbf{9}$ M/1-3 derechos; Glis glis 10 P/4 derecho; Talpa sp. 11 Húmero derecho, vista posterior; Crocidura russula 12a mandíbula derecha 12b cóndilo derecho; Sorex minutus 13a mandíbula izquierda 13b cóndilo izquierdo; Sorex araneus-coronatus 14a mandíbula izquierda; 14b cóndilo izquierdo. Barra de escala $1 \mathrm{~cm}$ para la figura 11 y $1 \mathrm{~mm}$ para el resto. / Some small mammal vertebrate fossil remains from Praileaitz I site (Deba, Gipuzkoa). Microtus (Microtus) arvalis $\mathbf{1}$ left M/1 2 left M/1; Microtus (Microtus) agrestis 3 left M/; Microtus (Terricola) sp. 4 right M/1; Chionomys nivalis $\mathbf{5}$ left M/1; Arvicola amphibius $\mathbf{6}$ right M/1-2; Clethrionomys glareolus 7a right M3/ 7b idem, lingual view; Microtus (Alexandromys) oeconomus 8 left M/1-2; Apodemus sylvaticus-flavicollis 9 right M/1-3; Glis glis 10 right P/4; Talpa sp. 11 right humerus, posterior view; Crocidura russula 12a right mandible 12b right condyle; Sorex minutus 13a left mandible 13b left condyle; Sorex araneus-coronatus 14a left mandible; 14b left condyle. Scale bars $=1 \mathrm{~cm}$ (figure 11) and $1 \mathrm{~mm}$ (figures $1-10$ and 12-14). 


\author{
Subgénero Terricola FATIO, 1867 \\ Microtus (Terricola) sp. FATIO, 1867 (Fig. 5.4)
}

Este subgénero está representado en todos los lechos del Pleistoceno. El M/1, tiene cuatro triángulos en el lado lingual y cinco en el labial. Los triángulos t4-t5, comunicados entre si, dibujan el llamado "primer rombo Pitimiano", mientras que los triángulos t6-t7 forman "el segundo triángulo Pitimiano".

En la actualidad coexisten en la Cornisa Cantábrica dos especies de este subgénero: $M$. (T.) lusitanicus y $M$. (T.) pyrenaicus, difíciles de difrenciar debido a su gran parecido morfológico. Por ende, optamos por determinar el material estudiado como $M$. (Terricola) sp. Son especies que viven en zonas de suelos profundos y húmedos, o bien en zonas de pradera con abundante vegetación.

\section{Subgénero Alexandromys OGnev, 1914} Microtus (Alexandromys) oeconomus (PALLAS, 1776) (Fig. 5.5)

El topillo nórdico, también conocido como Microtus ratticeps, habita en la actualidad en las zonas septentrionales de Eurasia, estando ausente en la Península Ibérica. Es una especie común en la Taiga y la Tundra, encontrándose en zonas muy húmedas como praderas, juncales y zonas pantanosas (SESÉ, 2005). Si bien es cierto que también aparece en las etapas climáticas más templadas, algunos autores (CHALINE, 1972; PEMÁN, 1985) relacionan el incremento de sus poblaciones durante el Pleistoceno con las etapas climáticas más frías.

\section{Género Chionomys MILLER, 1908 \\ Chionomys nivalis (MARTINS, 1842) (Fig. 5.6)}

Los molares de este taxón han sido hallados en el lecho solutrense y en el magdaleniense más antiguo de la primera sala interior y en la muestra magdaleniense más moderna del vestíbulo. En el M/1, de cuatro triángulos y entrantes en el lado lingual, y tres en el labial, el lóbulo anterior es muy característico, con una morfología de hongo inclinado hacia el lado labial.

Se encuentran poblaciones aisladas tanto en Europa como en Asia, mientras que en la Péninsula Ibérica habita en Sierra Nevada y los montes del norte. Es decir, en zonas de alta montaña, donde normalmente se encuentra en laderas orientadas al sur, en zonas abiertas y pedregosas de poca vegetación. De todas formas, KOWALSKI (1992) relaciona la presencia de este taxón más con la existencia de zonas abiertas que con zonas de alta montaña debido a la distribución presentada durante el Cuaternario.

\section{Género Arvicola LACÉPĖDE, 1799 \\ Arvicola amphibius (LINNAEUS, 1758) (Fig. 5.7)}

Tanto el lecho solutrense como los magdalenienses de la primera sala interior (muestras S1, S2 y S3) han proporcionado restos atribuibles a esta especie. Los M/1 presentan cuatro triángulos linguales, estando comunica- dos entre ellos el t4-t5. Por su parte, el complejo posterior esta constituido por tres triángulos no comunicados o débilmente comunicados entre ellos. A diferencia de su congénere $A$. sapidus, el esmalte es más grueso en el lado mesial de los triangulos (SDQ < 100).

La rata topera posee actualmente una amplia distribución paleártica, aunque en la Península Ibérica unicamente vive en poblaciones aisladas en el norte: desde los Pirineos hasta el norte de Portugal. Vive en alta montaña y en praderas, donde encuentra suelos profundos y húmedos para sobrevivir. También aparece cerca de los ríos y arroyos, pero nunca en bosques densos (SESÉ, 2005). En simpatría con $A$. sapidus, suele ocupar zonas más alejadas de los ríos y arroyos (CUENCA-BESCÓs et al., 2008).

\section{Arvicola cf. sapidus MILLER, 1908}

Un único M/1 hallado en el lecho superficial (muestra V1) ha dado un índice SDQ mayor de 100, por lo que a priori, lo separaría de A. amphibius. De todas maneras, al tratarse de un solo molar, que además no está muy bien preservado, la atribución del material a esta especie se realiza con reservas.

La rata de agua vive en las zonas húmedas, cerca de circulaciones constantes de agua y en zonas de vegetación herbácea y matorrales ubicados por toda la Península lbérica.

Género Clethrionomys TILESIUS, 1850 Clethrionomys glareolus SCHREBER, 1780 (Fig. 5.8)

Esta especie únicamente se registra en la muestra más moderna (muestra V1). Se caracteriza por la presencia de raíces y de cemento en los entrantes de los dientes, además de por tener un esmalte del mismo grosor en todo el perímetro oclusal.

Normalmente se asocia el topillo rojo a climas templados y zonas boscosas, o con abundante vegetación, aún cuando es posible encontrar esta especie en climas más fríos, aunque siempre en una menor proporción (PEMÁN, 1985, 1990).

Familia Muridae ILLIGER 1811

Subfamilia Murinae lLLIGER, 1811

Género Apodemus Kaup, 1829

Grupo Apodemus sylvaticus (LINNAEUS, 1758) -flavicoIlis (MELCHIOR, 1834) (Fig. 5.9)

Este grupo se encuentra representado en todas las muestras estudiadas, a excepción de los lechos magdalenienses del vestíbulo. La superficie oclusal de estos dientes braquiodontos está formada por seis cúspides en los primeros molares (tanto superiores como inferiores), y cuatro en los molares segundo y tercero (CUENCABESCÓS et al., 2008). Diferenciar entre las dos especies de este género que coexisten en la Península lbérica, $A$. sylvaticus y $A$. flavicollis, en función de las características de los molares no siempre resulta factible, por lo que 
se opta por agruparlas y describirlas dentro del grupo $A$. sylvaticus-flavicollis.

Es un grupo ampliamente repartido por Europa y la Península lbérica, si bien $A$. flavicollis posee una distribución más restringida, al habitar unicamente en la zona del norte. Por su parte, A. sylvaticus ocupa la totalidad de la Península lbérica, siendo más abundante en la zona mediterránea (ALCÁNTARA DE LA FUENTE, 1992). Consideradas como indicadores de climas cálidos y biotopos con zonas boscosas (PEMÁN, 1990), es posible identificar restos de este grupo tanto en periodos templados, como en más frios del Pleistoceno y Holoceno de la Cornisa Cantábrica. Aún así, las poblaciones suelen ser más abundantes cuanto más benignas son las condiciones ambientales, con temperaturas suaves, humedad alta y desarrollo importante de masas boscosas.

Familia Gliridae MUIRHEAD, 1819

Género Glis Brisson, 1762

Glis glis (LINNAEUS, 1766) (Fig. 5.10)

El único molar hallado en el lecho superficial (muestra V1) es atribuido a esta especie debido a la complejidad de la morfología dentaria, caracterizado por las crestas accesorias.

Especie asociada a bosques cálidos, en el País Vasco únicamente habita en bosques caducifolios. Al no identificarse en los niveles fríos del Pleistoceno superior y Holoceno inferior, la presencia del lirón gris es considerada como indicativa de clima templado y masas arbóreas importantes (PEMÁN, 1985).

Orden EULIPOTYPHLA WADDELL, OKADA Y HASEGAWA, 1999 Familia Talpidae FISCHER, 1814.

Género Talpa LinNAEUs, 1758

Talpa sp. (Fig. 5.11)

Los lechos solutrenses y magdalenienses de la primera sala interior han proporcionado registro de este taxón, identificado por el tamaño de los molares y un esqueleto postcraneal muy característico. La falta de material hace que no se pueda precisar más allá del nivel de género.

Talpa es un género muy extendido en Europa, aunque en la Península Ibérica unicamente aparece en el noreste del territorio, llegando hasta el sistema Central en el sur (SESÉ, 2005). Los topos se relacionan con praderas humedas con suelos profundos y fáciles de excavar, por lo que la presencia del género puede tomarse como indicativo de humedad (CUENCA-BESCÓs et al., 2008).

Familia Soricidae FISCHER, 1814

Subfamilia Crocidurinae MILNE-EDWARDS, 1872.

Género Crocidura WAGLER, 1832.

Crocidura russula HERMANN, 1870 (Fig. 5.12)

Documentada en el Epipaleolitico y en la muestra más superficial de Praileaitz I, la asignación a esta es- pecie se basa en la morfología del cíngulo en los $\mathrm{M} / 1,2$ y del M/1, además de la forma subtriangular del cóndilo y la ausencia de coloración en los dientes propios del género.

La musaraña gris vive en biotopos secos y descubiertos, aunque también se puede encontrar en bosques y zonas arbustivas (SESÉ, 2005; PEMÁN, 1985).

Subfamilia Soricinae FISCHER, 1814

Género Sorex LINNAEUS, 1758

Sorex minutus LINNAEUS, 1766 (Fig. 5.13)

El pequeño tamaño de las piezas estudiadas, y la coloración rojiza de los dientes, hacen que el material se incluya dentro de la variación existente en el grupo $S$. minutus.

La musaraña enana vive en zonas con densa cobertera vegetal, en pastizales y bosques, asociada sobre todo a ambientes húmedos y fríos (SESÉ, 2005).

Grupo Sorex araneus LINNAEUS, 1758 - coronatus MILLET, 1882 (Fig. 5.14)

Este grupo está representado en todos los lechos crono-culturales. Los incisivos inferiores, con el color rojo propio de la subfamilia, presentan tres cúspulas en la superficie oclusal.

Tanto la musaraña bicolor, como la tricolor, habitan en la zona norte de la Península Ibérica, aunque $S$. araneus lo hace desde los Pirineos hasta el Prepirineo Catalán, y S. coronatus desde los Pirineos hasta Galicia (LÓPEZ-FUSTER y VENTURA, 1996). Se encuentran en zonas húmedas con abundante vegetación tipo herbácea, arbustiva o arbórea (POKINES, 1998; CUENCABESCÓs et al., 2008).

Clase AMPHIBIA GrAY, 1825

Orden ANURA FISCHER VON WALDHEIM, 1813

Familia Alytidae FITZINGER, 1843

Género Alytes WAGLER, 1829

Alytes cf. obstetricans (LAURENTI, 1768) (Fig. 6.1)

El lecho Solutrense de la primera sala interior ha proporcionado una tibia-fíbula y un ilion asignables a esta especie debido al marco geográfico. El ilion, desprovisto de ala dorsal, presenta una pars ascendens que, aunque rota, es claramente alargada. La tuberosidad superior está bien desarrollada, situada sobre gran parte de la cavidad acetabularia, pero sin sobresalir lateralmente.

El sapo partero común, abundante en el tercio norte peninsular, se distribuye principalmente por zonas de alta pluviosidad, ya que requiere de puntos de agua casi permanentes a causa de su prolongado desarrollo larvario. Normalmente está relacionado con praderas húmedas, donde se entierra a bastante profundidad. Debido a esta habilidad, su presencia en algunos yacimientos arqueológicos puede deberse a muertes in situ (GARCÍA-PARÍS et al., 2004; PLEGUEZUELOS et al., 2004). 

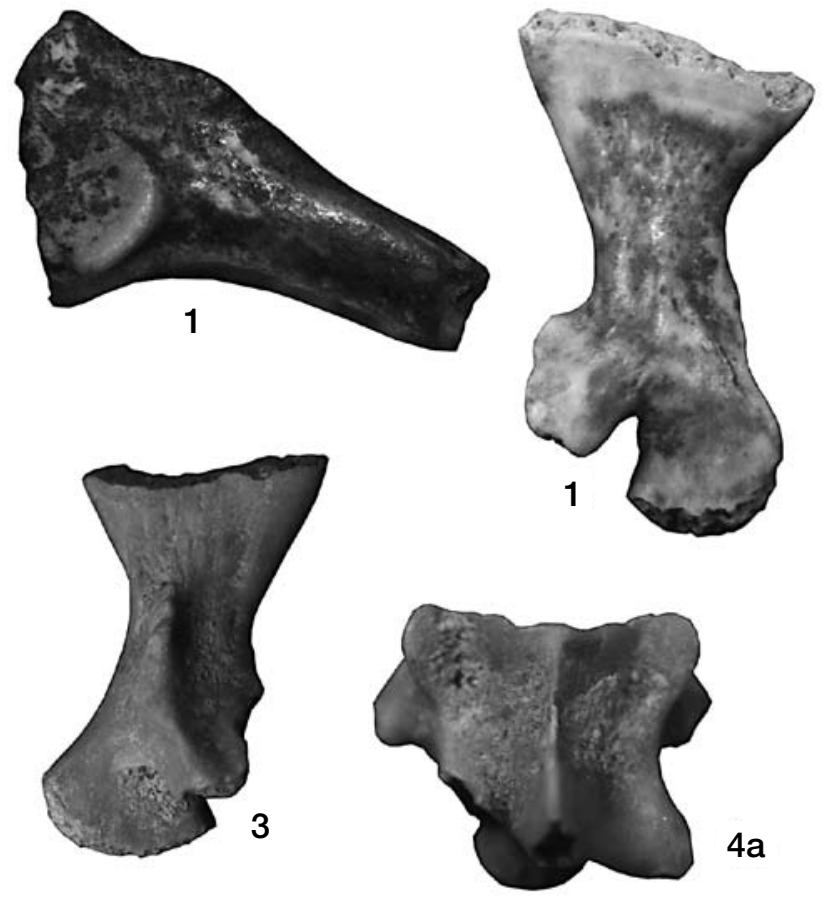

$4 a$

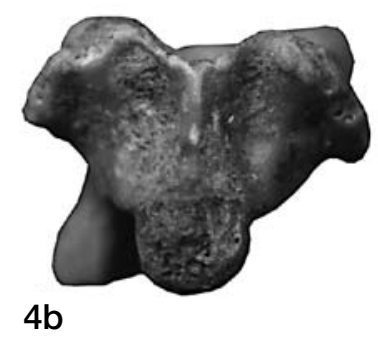

$4 b$

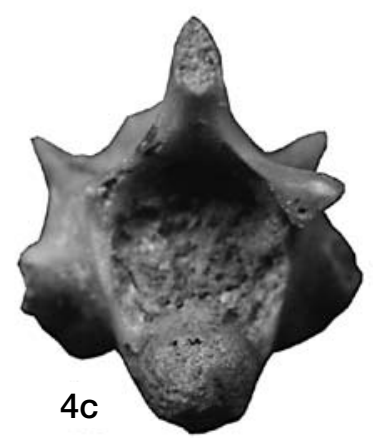

Fig. 6. Algunos de los restos de anfibios y reptiles identificados en el yacimiento de Praileaitz I (Deba, Gipuzkoa). Alytes cf. obstetricans 1 ilion derecho; Bufo bufo 2 escapula izquierda; Rana temporaria-iberica $\mathbf{3}$ escapula derecha; cf. Lacerta 4a-c vértebra dorsal vista dorsal, ventral y posterior. Barra de escala $0.5 \mathrm{~mm}$ para las figuras 1-2 y $1 \mathrm{~mm}$ para las figuras 3-4. / Some amphibians and reptiles fossil remains from Praileaitz I site (Deba, Gipuzkoa). Alytes cf. obstetricans 1 right ilium; Bufo bufo 2 left scapula; Rana temporaria-iberica $\mathbf{3}$ rigth scapula; cf. Lacerta $\mathbf{4 a - c}$ trunk vertebrae in dorsal, ventral and posterior view. Scale bars $=0.5 \mathrm{~mm}$ (figures $1-2$ ) and $1 \mathrm{~mm}$ (figures 3-4).
Familia Ranidae RAFINESQUE-SCHMALTZ, 1814

Género Rana LINNAEUs, 1758

\section{Grupo Rana temporaria LINNAEUS, 1758 - iberica BOULEN-} GER, 1879 (Fig. 6.2)

Aun cuando no está presente en todas las muestras analizadas, es el anuro dominante en Praileaitz I. Los restos de este grupo provienen de los lechos Solutrense y Magdaleniense de la primera sala interior y del vestíbulo. A partir del análisis osteológico, se descarta que los restos de Praileaitz I pertenezcan al subgénero Pelophylax, aunque como no es posible especificar más, optamos por denominar los restos dentro del grupo Rana temporaria-iberica. La escápula es alargada y aplanada, con la característica cresta glenoidea encorvada que llega hasta el borde anterior del hueso atípica de las ranas pardas (BAILON, 1999). En el ilion, de cresta dorsal baja e inclinada medialmente, el ratio diámetro acetabular vs. grosor (d/t; sensu GLEED-OWEN, 2000) proporciona unos valores (3.2-3.4) que entran dentro del rango de las ranas pardas (2.75-4).
Mientras que la rana ibérica es endémica de la Península Ibérica, la rana bermeja es una especie eurosiberiana muy abundante en la región Paleártica. La rana ibérica está asociada a arroyos y regatos de aguas frías y rápidas con abundante vegetación en las orillas. Por su parte, la rana bermeja presenta hábitos bastante terrestres, siendo frecuente que emigre hacia zonas de matorral y bosques de hayas y robles (GARCÍA-PARís et al., 2004; PLEGUEZUELOS et al., 2004).

Familia Bufonidae GrAY, 1825

Género Bufo LAURENTI, 1768

Bufo bufo (LINNAEUS, 1758) (Fig. 6.3)

Es la segunda especie de anfibio en importancia cuantitativa en Praileaitz I, habiéndose identificado en 3 muestras magdalenienses (tanto del vestíbulo, como de la primera sala interior). Entre las piezas óseas identificadas destacan un único ilion, una escápula y una vértebra sacra con morfología típica de bufónido. La atribución a Bufo bufo está basada en la presencia sobre el ilion de 
una tuberosidad superior simple y con borde dorsal redondeado. Por su parte, la escapula es alargada, con un borde anterior sinuoso y una apófisis glenoidea bien diferenciada del resto de hueso, y carece de fosa supraglanoidea. En cuanto a la vértebra sacra, las apófisis están moderadamente expandidas y carece de fosa dorsal (SANCHIZ, 1977; BAILON, 1999).

El sapo común está presente en toda la Península Ibérica. Aunque ocupa todo tipo de hábitats, su presencia puede estar condicionada por la existencia de cursos de agua constantes y de cierta profundidad, preferentemente con vegetación. En el País Vasco prefiere los bosques caducifolios con ríos y torrentes (GARCÍA-PARíS et al., 2004; PLEGUEZUELOS et al., 2004).

Clase REPTILIA LAURENTI, 1798

Orden SQUAMATA OPPEL, 1811

Familia Lacertidae OPPEL, 1811

Género cf. Lacerta LinnAEUS, 1758 (Fig. 6a-c)

Disponemos de una única vértebra dorsal asignable a esta especie. Esta vértebra, procela y con cótilo y cóndilo circulares, tiene un cuerpo neural corto y presenta una carena hemal prominente pero fina. Este conjunto de caracteres permiten atribuir la vértebra a un lacértido. La talla de la vértebra es comparable a la de un representante del género Lacerta, pero no es posible avanzar una atribución más específica.

\section{5.- PALEOECOLOGÍA}

La curva de la variación relativa de la temperatura (Fig. 7) se ha estimado en base al Número Mínimo de Individuos (NMI) de cada uno de los taxones de micromamíferos, su proporción relativa en la muestra estudiada (Tabl. 3) y su afinidad ecológica (Tabl. 2). Si observamos la curva de la primera sala interior, vemos que las condiciones ambientales son frías para el Solutrense (muestra S3) y el Magdaleniense Inferior (muestras S2 y S3), aun- que se da una pequeña variación hacia condiciones más cálidas en la muestra más antigua del Magdaleniense Inferior (muestra S2). Por su parte, las muestras del vestíbulo siguen durante el Magdaleniense Inferior (muestras V7 y V6) la misma dinámica que en la primera sala interior, con unas condiciones ambientales frías. Las muestras correspondientes al Epipaleolítico (muestras V5, V4 y V3) indican un fuerte calentamiento, aunque no hay que olvidar que, como se ha comentado en el apartado de tafonomía, estas muestras son muy pobres y han sufrido unas alteraciones tafonómicas particulares que pueden haber alterado los restos. En el lecho superficial (muestras V2 y V1), las condiciones ambientales son cálidas y similares a las observadas en los registros holocénicos de la Cornisa Cantábrica.

Debido a que la mayoría de los restos de herpetofauna corresponden a Bufo bufo y Rana temporariaiberica, el paleoambiente sugerido por estos taxones para el entorno de Praileaitz I correspondería al de un medio dominado por bosques caducifolios, con alguna zona abierta de pradera húmeda cercana a algún punto de agua como ríos o estanques permanentes. Es cierto que Alytes obstetricans es una especie que prefiere los biotopos soleados y abiertos, pero su presencia en este yacimiento está limitada a un único individuo. De todo ello se deduce que probablemente nos encontraríamos ante una situación climática más húmeda que la actual.

En cuanto a las variaciones entre los lechos, la muestra V7 correspondería a un momento de escasa humedad, mientras que el S3 representaría el momento más húmedo y menos boscoso de todos, con claro predomino de las ranas.

\section{6.- CONCLUSIONES}

Se han identificado 13 taxones diferentes de micromamíferos (Microtus (Microtus) arvalis, Microtus (Microtus) agrestis, Microtus (Terricola) sp., Microtus (Alexan-

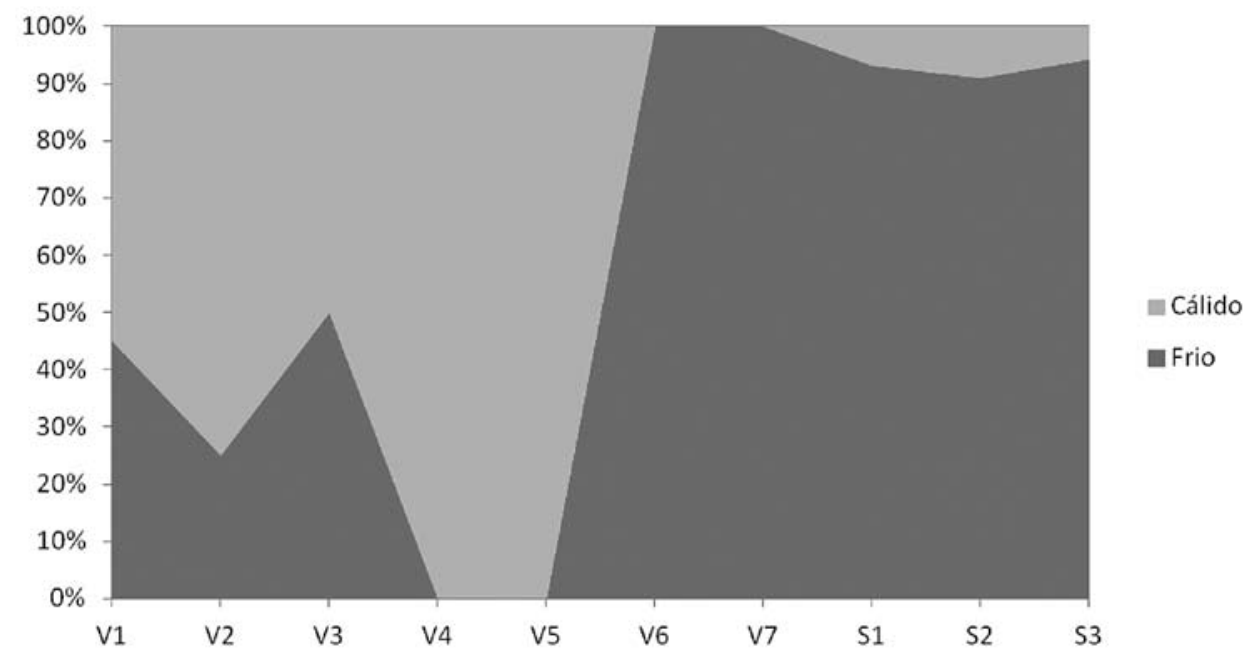

Fig. 7. Variación relativa de la temperatura en base a las afinidades autoecológicas de los diferentes taxones de micromamíferos presentes en las muestras del yacimiento de Praileaitz I (Deba, Gipuzkoa). / Relative variation of the temperature based on the autoecological affinities of the different taxa of small mammals identified in the samples from Praileaitz I site (Deba, Gipuzkoa). 
dromys) oeconomus, Chionomys nivalis, Arvicola amphibius, Arvicola cf. sapidus, Clethrionomys glareolus, Apodemus sylvaticus-flavicollis, Glis glis, Talpa sp., Crocidura russula, Sorex minutus y Sorex araneus-coronatus), tres anfibios (Alytes cf. obstetricans, Bufo bufo y Rana temporaria-iberica) y un reptil (cf. Lacerta).

Se ha realizado el estudio de los microvertebrados obtenidos con el tamiz de 0,5 mm de luz de malla, habiéndose recuperado un total de 82573 elementos. Tambien han sido estudiados los 1089 restos obtenidos con el tamiz de más de $2 \mathrm{~mm}$.

La conservación de los diferentes elementos anatómicos indica que las muestras V3, V4 y V5 son las que han sufrido una mayor alteración tafonómica. La cantidad de restos del lecho V6 es muy superior al resto de las muestras. Este dato podría indicar una mayor actividad de las rapaces en ese momento, y por tanto, ausencia de actividad humana permanente. La distribución de restos en la superficie excavada nos indica que mientras en el vestíbulo parece que los restos se encuentran acumulados en ciertos cuadros, en las salas interiores la distribución es más aleatoria. Estos datos pueden interpretarse como la acumulación de los restos en el vestíbulo por algún agente biológico, posiblemente una rapaz, y escaso o nulo transporte de los mismos antes de su enterramiento. En las salas interiores los restos podrían haber sido transportados por algún flujo tractivo, como una suave corriente de agua, y puede que en menor medida también por grandes mamíferos.

Las afinidades ecológicas de los micromamíferos preservados indican que durante el depósito de los lechos más antiguos (Solutrense y Magdaleniense Inferior) las condiciones ambientales fueron las más frías de las registradas en toda la serie, mientras que en los lechos superiores se pasa a unas condiciones relativamente más cálidas. Los lechos V3, V4 y V5 (Epipaleolítico) presentan unas condiciones muy cálidas, aunque esto puede ser efecto de la alteración tafonómica de los restos.

\section{7.- AGRADECIMIENTOS}

Agradecemos a Xabier Peñalver el habernos ofrecido la posibilidad de estudiar la microfauna de Praileaitz I y a todo el equipo de excavación el habernos recuperado las muestras para nuestro estudio. Así como, a Ana Uriz y Pablo Areso el haber puesto a nuestra disposición los residuos de su estudio. También agradecer a Nagore Pérez su ayuda en el pesado de las muestras. Aitziber SuárezBilbao es receptora de una beca de formación de investigadores (PRE_2014_1_345) del Gobierno Vasco. Por su parte, Juan Rofes cuenta con un contrato Marie Curie Fellowship (MCA-IEF Project n629604) de la Comisión Europea. Los firmantes de este trabajo forman parte del Grupo de Investigación de la Universidad del País Vasco GIU12/35 y del proyecto PALEOGATE del Ministerio de Ciencias del Gobierno de España (HAR2014-53536-P).

\section{8.- BIBLIOGRAFÍA}

ALCÁNTARA DE LA FUENTE, M.

1992 Distribución y preferencias de hábitat de los micromamíferos (Insectivora y Rodentia) de la Sierra de Guadarrama. Tesis doctoral. Inédita. Universidad Complutense de Madrid. Facultad de Ciencias Biológicas. Departamento de Biología animal I (Zoología de Vertebrados).

ANDREWS, P.

1990 Ow/s, Caves and Fossils. Natural History Museum Publications. London.

ANDREWS, P., EVANS, E.M.N.

1983 Small mammal bone accumulations produced by mammalian carnivores. Paleobiology 9 (3), 289-307.

ARRIZABALAGA, A., MONTAGUT, E., GOSÁLBEZ, J.

1986 Introducció a la Biologia i zoogeografia dels petits mamífers (insectivors i rosegadors) del Montseny (Catalunya). CIRIT. Barcelona.

BAlLON, S.

1991 Amphibiens et reptiles du Pliocène et du Quaternaire de France et d'Espagne mise en place et évolution des faunes. Thèse 3ème cycle. Université de Paris VII, France.

1999 Clés de détermination ostéologique des amphibiens anoures de France, en DESSE, J. \& DESSE BERSET, N. (eds). Fiches d'ostéologie animale pour I 'Archéologie, Varia. 1-38. Centre de Recherches Archéologiques du CNRS. Paris.

BLAIN, H.A.

2009 Contribution de la paléoherpétofaune (Amphibia \& Squamata) à la connaissance de l'évolution du climat et du paysage du Pliocène supérieur au Pléistocène moyen d'Espagne. Treballs del Museu de Geologia de Barcelona 16, 39-170.

CARRETERO, M.A., AYLLÓN, E., LLORENTE, G.

2011 Lista patrón actualizada de los anfibios y reptiles de España. Disponible en: wuw.herpetologica.es/attachments/ article/112/Nueva Lista Patrón 2011.pdf.

CHALINE, J.

1972 Les Rongeurs du Pléistocène Moyen et Supérieur de France. Systématique, Biostratigraphie, Paléoclimatologie. C.N.R.S. Paris.

CUENCA-BESCÓS, G., STRAUS, L. G., GONZÁLEZ MORALES, M.R., GARCÍA PIMIENTA, J.C

2008 Paleoclima y paisaje del final del Cuaternario en Cantabria: Los pequeños mamíferos del Mirón (Ramales de la Victoria). Revista Española de Paleontología 23 (1), 91-126.

DAAMS, R

1981 The dental pattern of the dormice Dryomys, Myomimus, Microdyromys and Peridyromys. Tesis doctoral. University of Utrech. 
GARCÍA-PARÍS, M., MONTORI, A., HERRERO, P.

2004 Amphibia. Lissamphibia, en RAMOS, M.A. et al. (eds.). Fauna Ibérica, vol. 24. Museo Nacional de Ciencias Naturales. Madrid.

GLEED-OWEN, C.P.

2000 Subfossil records of Rana cf. lessonae, Rana arvalis and Rana cf. dalmatina from Middle Saxon (c. 600-950 AD) deposits in eastern England: evidence for native status. Amphibia-Reptilia 21, 57-65.

HEINRICH, W.D.

1978 Biometrischen Erfassung eines Evolutionstrends bei Arvicola (Rodentia, Mammalia) aus dem Pleistozän Thüringens. Säugetierkunde Informationen 2, 3-21.

1982 Ein Evolutionstrend bei Arvicola (Rodentia, Mammalia) und seine Bedeutung für die Biostratigraphie im Pleistozän Europas. Wissenschaftliche Zeitschrift der Humboldt-Universität zu Berlin. Mathematisch-Naturwissenschaftliche Reihe, XXXI (3), 155-160.

KOWALSKI, K.

1992 Remarks on the zoogeography of Quaternary Rodents in Europe. Courier Forschungsinstitut Senckenberg 153 197-203.

LÓPEZ-FUSTER, M.J., VENTURA, J.

1996 A morphometrical review of the Sorex araneus-articus species group from the Iberian Peninsula (Insectivora, Soricidae). Bonner Zoologische Beiträge 46, 327-337.

PEMÁN, E.

1985 Aspectos climáticos y ecológicos de los micromamíferos del yacimiento de Erralla. Munibe Antropologia-Arkeologia 37, 49-57.

1990 Los micromamíferos en el Pleistoceno superior del País Vasco. Munibe Antropologia-Arkeologia 42, 259-262.

PINTO-LLONA, A.C., ANDREWS, P.

1999 Amphibian taphonomy and its application to the fossil record of Dolina (middle Pleistocene, Atapuerca, Spain). Palaeogeography, Palaeoclimatology, Palaeoecology 149, 411-429.

PLEGUEZUELOS, J.M., MÁRQUEZ, R., LIZANA, M.

2004 Atlas y libro rojo de los Anfibios y Reptiles de España. Dirección General de Conservación de la Naturaleza-Asociación Herpetológica Española. Madrid.
POKINES, J. T.

1998 The paleoecology of Lower Magdalenian Cantabrian Spain. Oxford. (Bar International series, 713).

REUMER, J. W. F.

1984 Rusnician and early Pleistocene Soricidae (Insectivora, Mammalia) from Tegelen (The Netherlands) and Hungary. Scripta Geologica 73, 1-173.

ROFES, J., ZULUAGA, M.C., MURELAGA, X., FERNÁNDEZERASO, J., BAILON, S., IRIARTE, M.J., ORTEGA, L.A., ALONSOOLAZABAL, A.

2013 Paleoenvironmental reconstruction of the early Neolithic to middle Bronze Age Peña Larga rock shelter (Álava, Spain) from the small mammal record. Quaternary Research 79 (2), 158-167.

\section{SALVADOR, A. (COORD.)}

1998 Reptiles, en RAMOS, M.A. et al. (eds.). Fauna Ibérica, 10. Museo Nacional de Ciencias Naturales. Madrid.

\section{SANCHIZ, B.}

1977 La familia Bufonidae (Amphibia, Anura) en el Terciario Europeo. Trabajos sobre Neogeno-Cuaternario 8, 75-111.

SESÉ, C.

2005 Aportación de los micromamíferos al conocimiento paleoambiental del Pleistoceno Superior de la Región Cantábrica: nuevos datos y síntesis. Monografía del Museo Nacional y Centro de investigación de Altamira 20, 167200.

TESAKOV, A., LEBEDEV, V.S., BANNIIKOVA, A.A., ABRAMSON, N.I.

2010 Clethrionomys Tilesius, 1850 is the valid generic name for red-backed voles and Myodes Pallas, 1811 is a junior synonym of Lemmus Link, 1795. Russian Journal of Theriology 9, 83-86.

VAN DER MEULEN, A.

1973 Middle Pleistocene smaller mammals from Monte Peglia (Orvieto, Italy) with special reference to the phylogeny of Microtus (Arvicolidae, Rodentia). Quaternaria 17, 1-144.

WILSON, D.E., REEDER, D.M .

2005 Mammal Species of the World. A Taxonomic and Geographic Reference. John Hopkins University Press. Baltimore. 
ANEXOS

Anexo l.l. Vestíbulo

Estimación de la distribución de los restos de microvertebrados en la zona del Vestíbulo del yacimiento de Praileaitz I (Deba, Gipuzkoa). I Estimation of the distribution of the microvertebrates at the Vestible of Praileaitz I site (Deba, Gipuzkoa).

Lecho 1

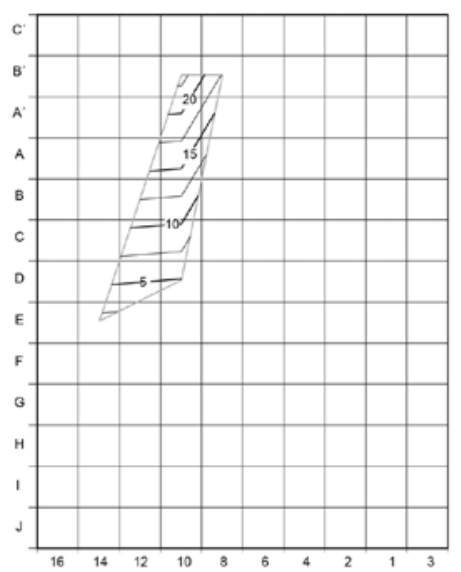

Lecho 4

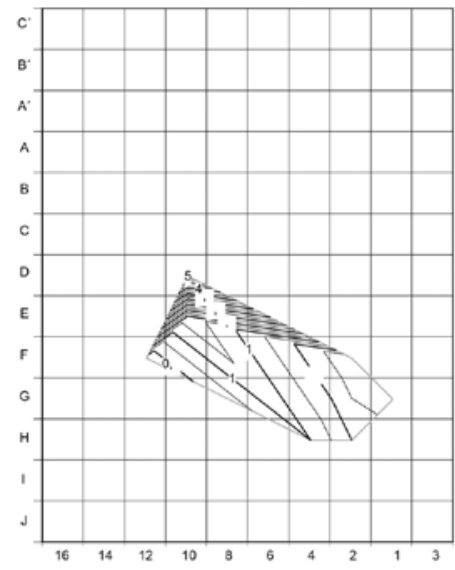

Lecho 7

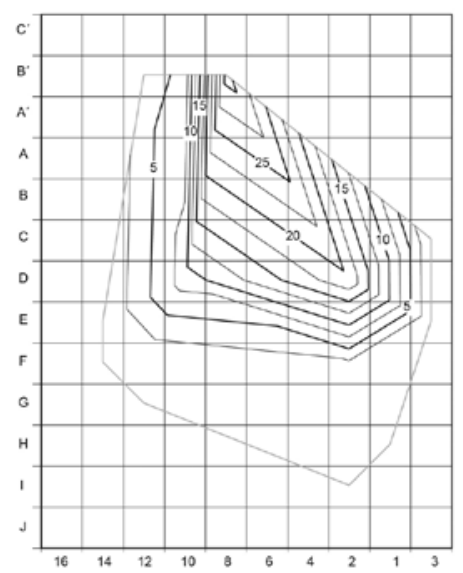

Lecho 2

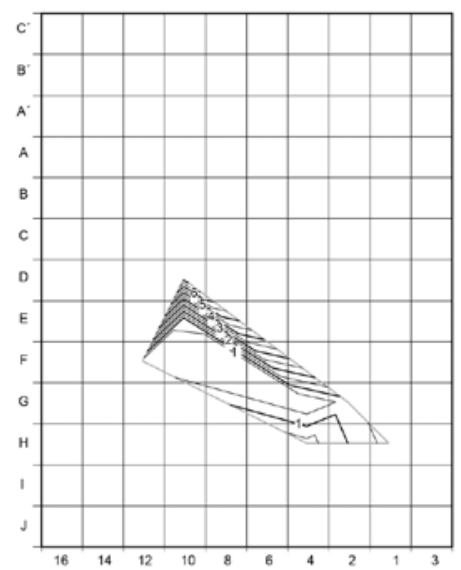

Lecho 5

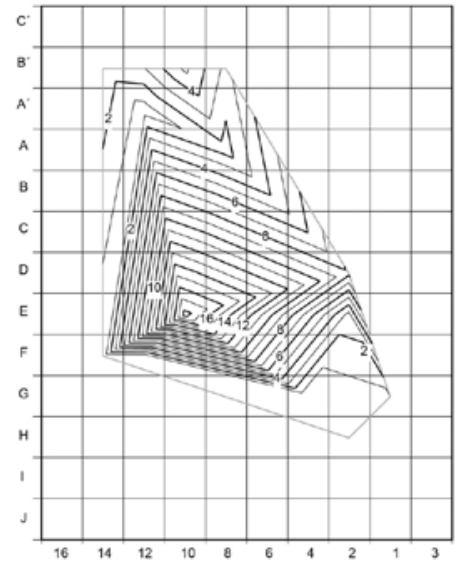

Lecho 8

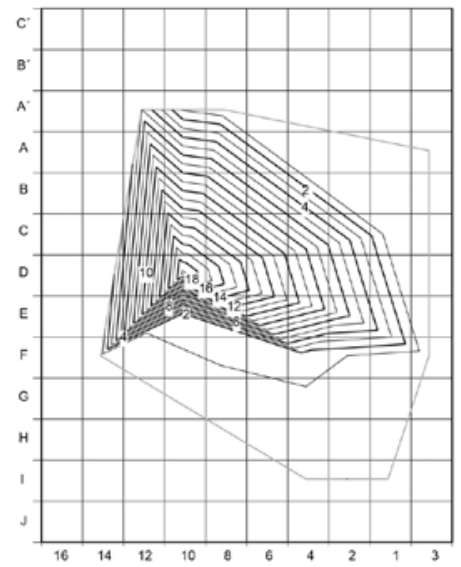

Lecho 3

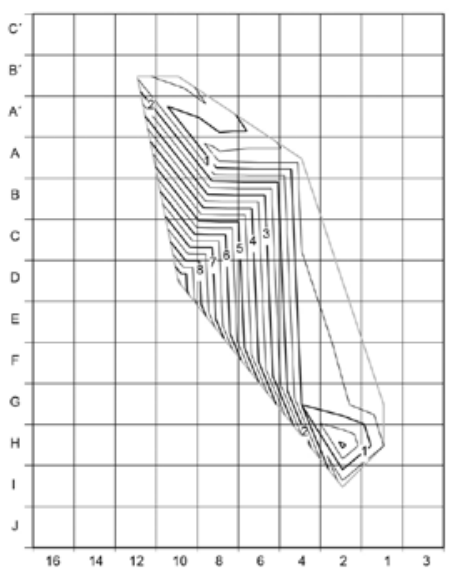

Lecho 6

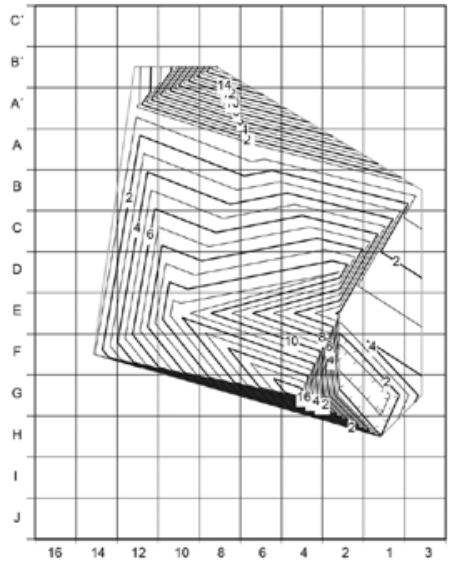

Lecho 9

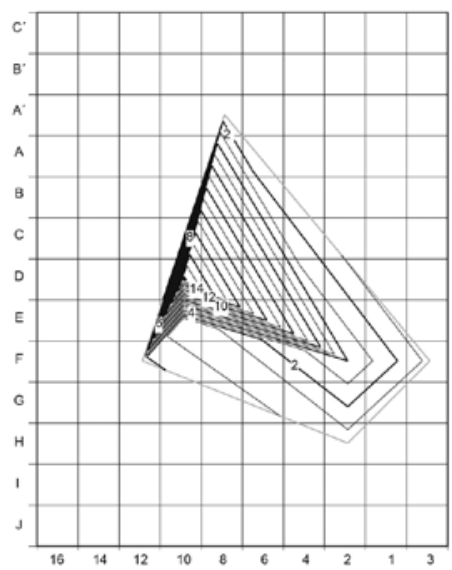




\section{Anexo I.II. Vestíbulo}

Estimación de la distribución de los restos de microvertebrados en la zona del Vestíbulo del yacimiento de Praileaitz I (Deba, Gipuzkoa). / Estimation of the distribution of the microvertebrates at the Vestible of Praileaitz I site (Deba, Gipuzkoa).
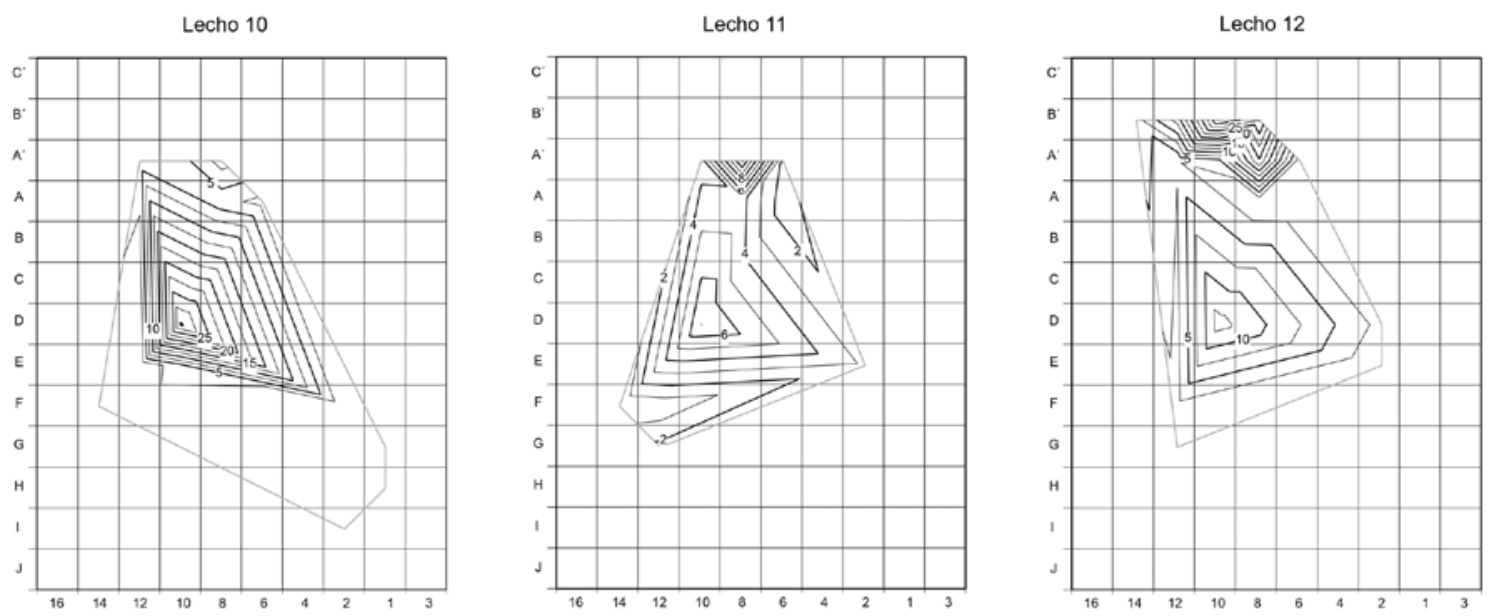

Lecho 13

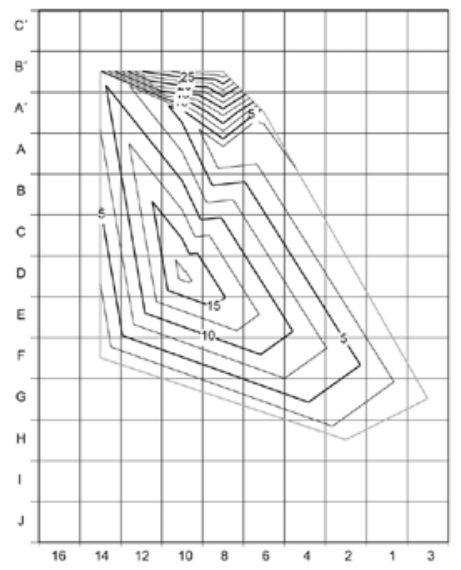

Lecho 14

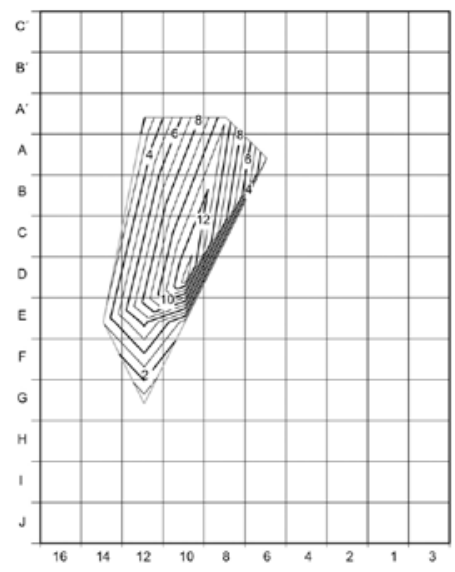

Lecho 15

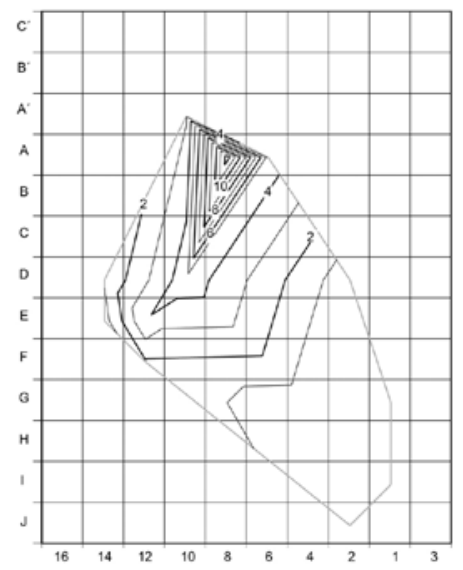

Lecho 16

Lecho 17
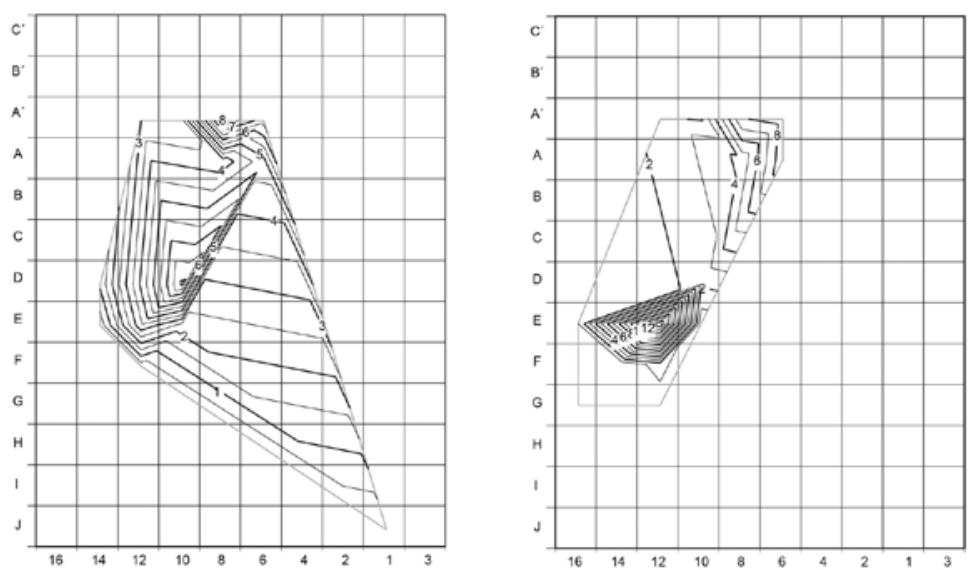

Lecho 18

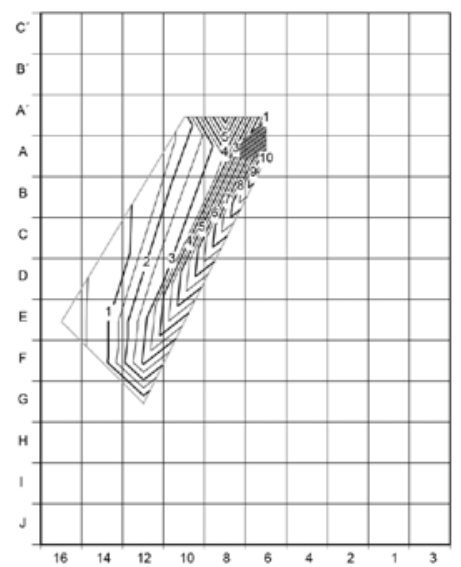




\section{Anexo I.III. Vestíbulo}

Estimación de la distribución de los restos de microvertebrados en la zona del Vestíbulo del yacimiento de Praileaitz I (Deba, Gipuzkoa). I Estimation of the distribution of the microvertebrates at the Vestible of Praileaitz I site (Deba, Gipuzkoa).

Lecho 19

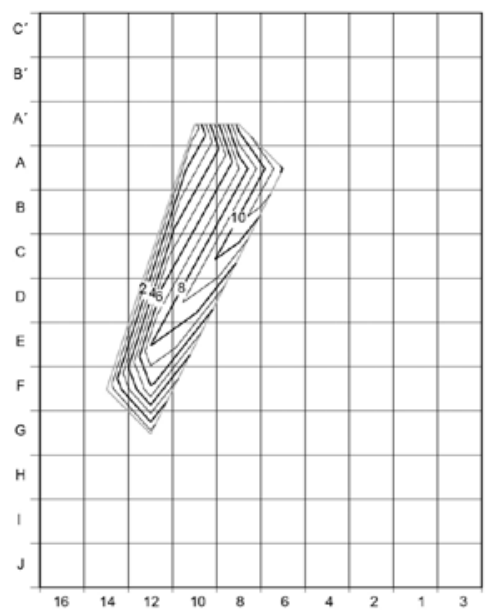

Lecho 22

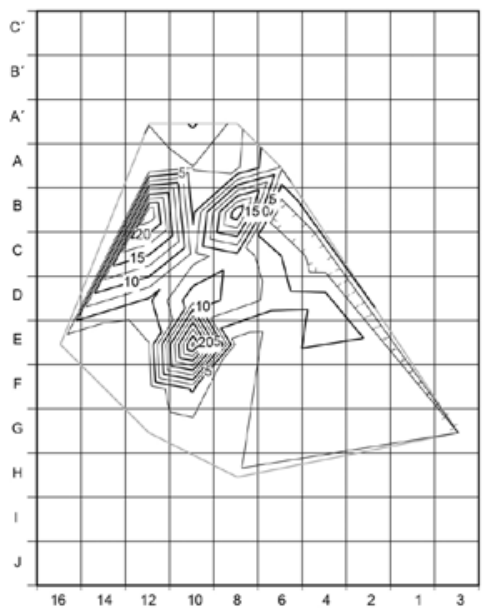

Lecho 25

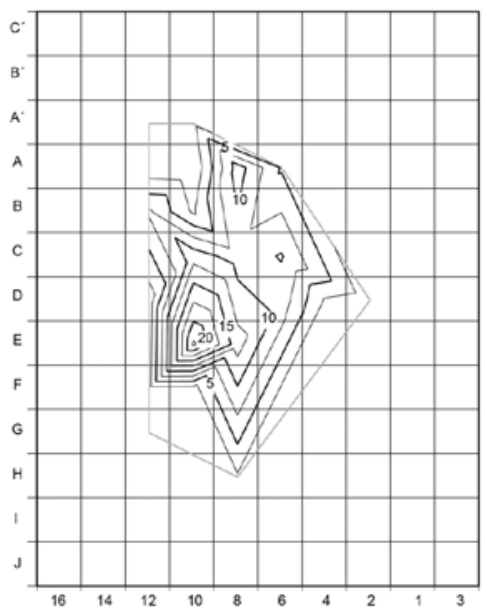

Lecho 20

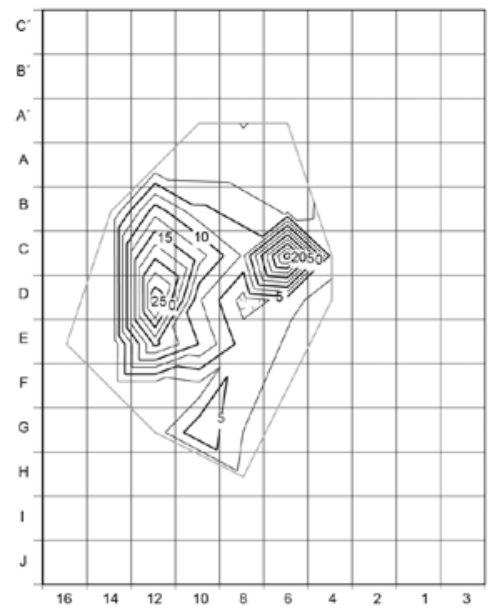

Lecho 23

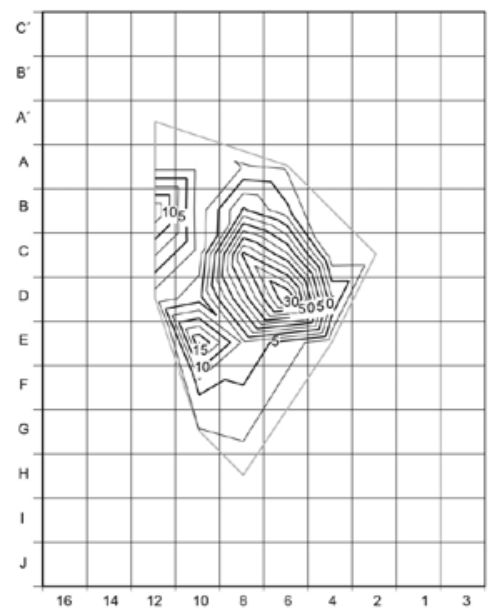

Lecho 26

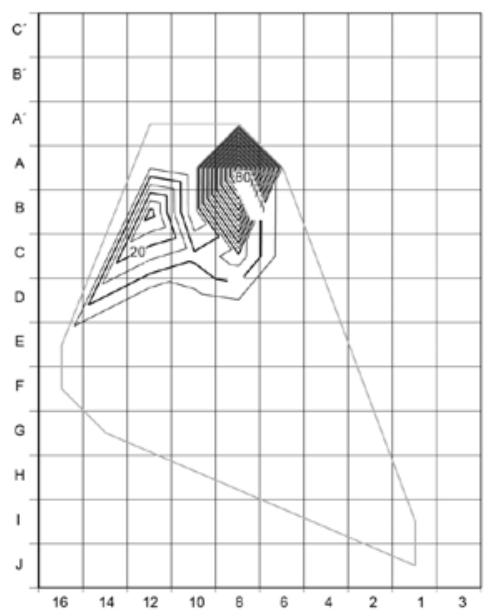

Lecho 21

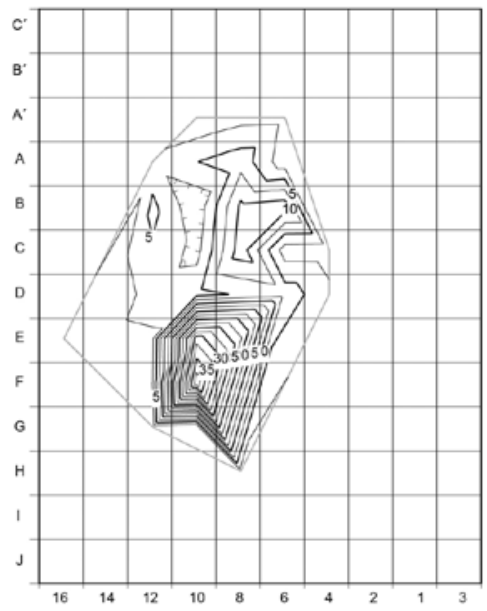

Lecho 24

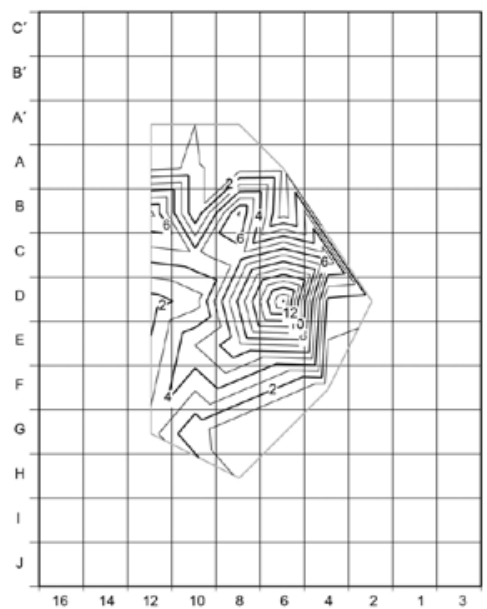




\section{Anexo II.I. Primera Sala interior}

Estimación de la distribución de los restos de microvertebrados en la $1^{\circ}$ Sala interior del yacimiento de Praileaitz I (Deba, Gipuzkoa). I Estimation of the distribution of the microvertebrates at the 1st Interior Chamber of Praileaitz I site (Deba, Gipuzkoa).

Lecho 2

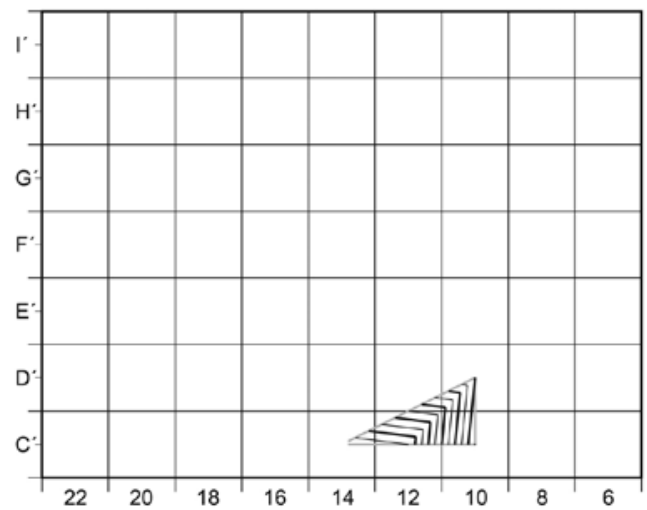

Lecho 5

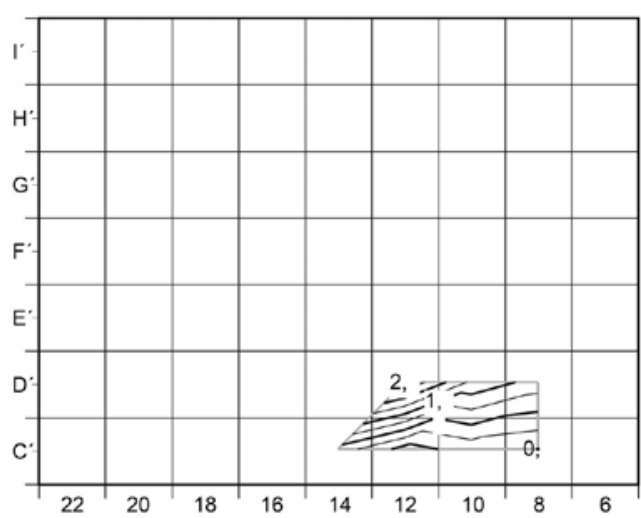

Lecho 7

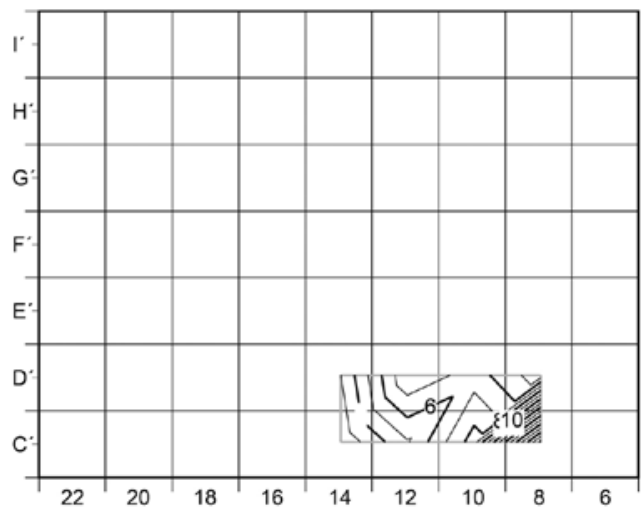

Lecho 4

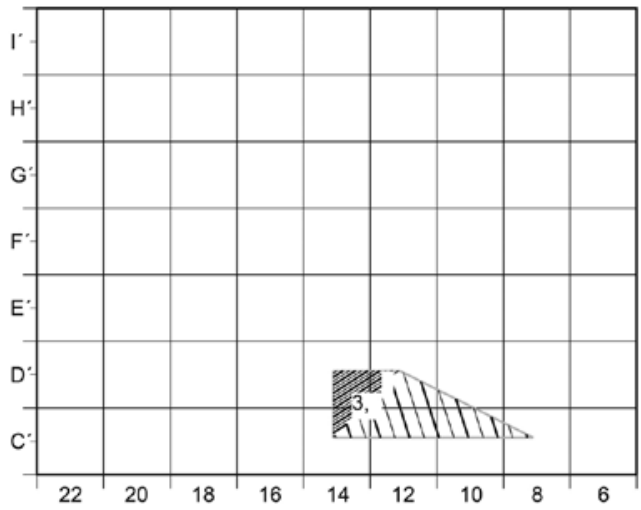

Lecho 6

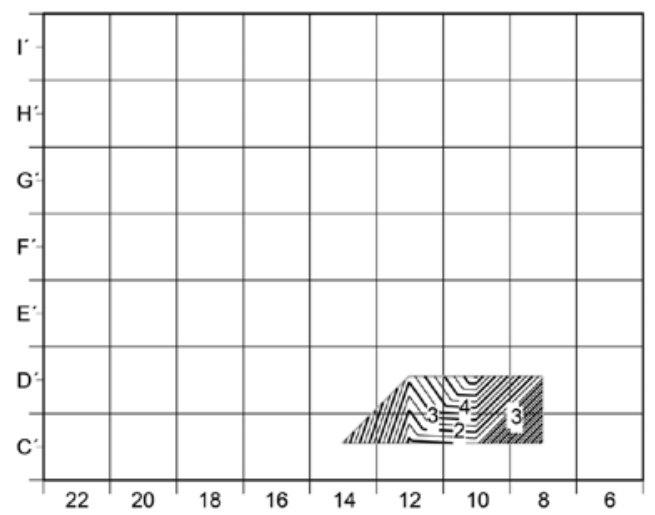

Lecho 9

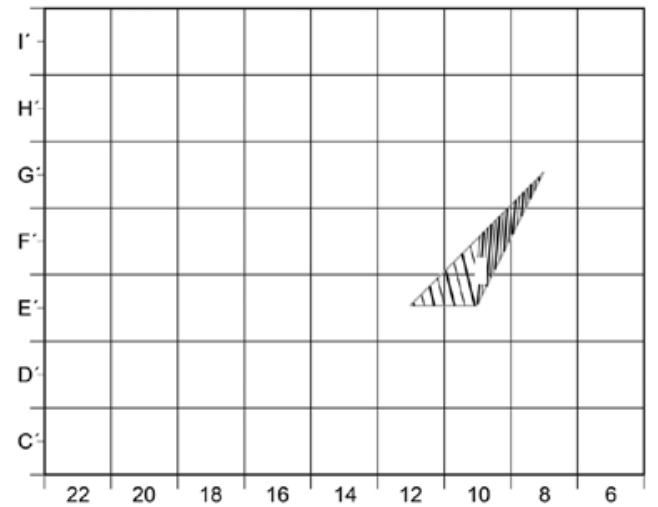




\section{Anexo II.II. Primera Sala interior}

Estimación de la distribución de los restos de microvertebrados en la $1^{\circ}$ Sala interior del yacimiento de Praileaitz I (Deba, Gipuzkoa). / Estimation of the distribution of the microvertebrates at the 1st Interior Chamber of Praileaitz I site (Deba, Gipuzkoa).

Lecho 10

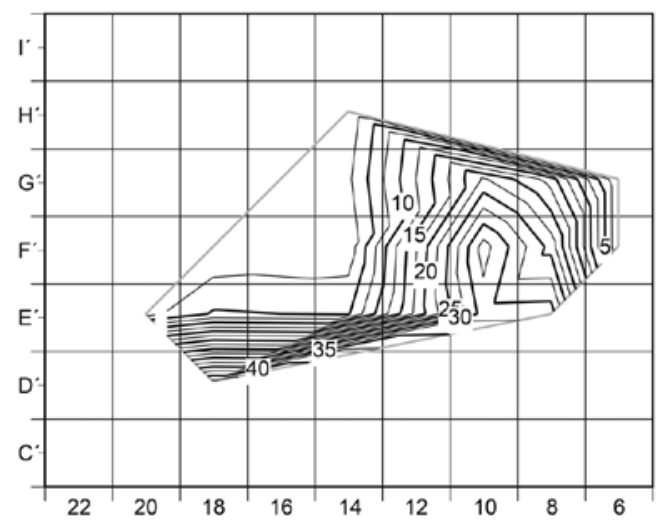

Lecho 12

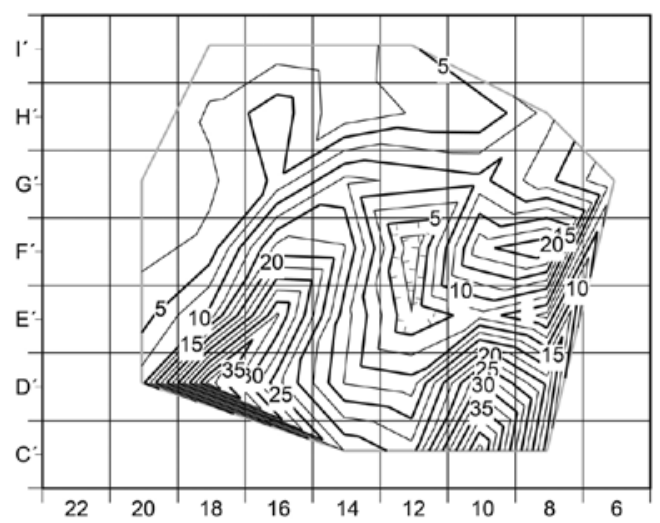

Lecho 11

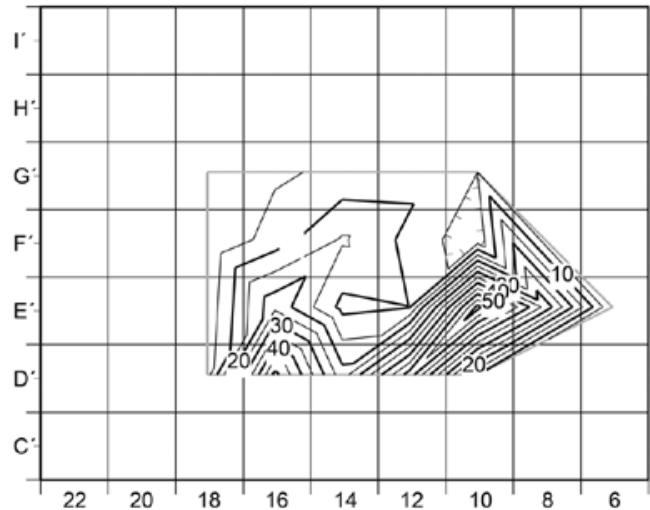

Lecho 13

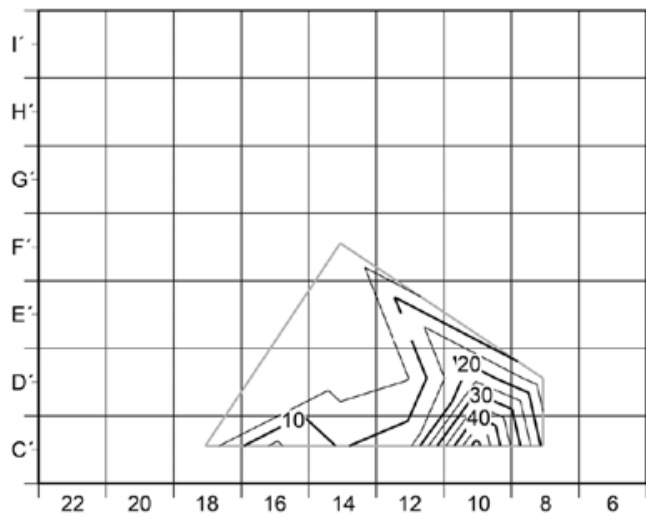




\section{Anexo III. Segunda Sala interior}

Estimación de la distribución de los restos de microvertebrados en la $2^{\circ}$ Sala interior del yacimiento de Praileaitz I (Deba, Gipuzkoa). / Estimation of the distribution of the microvertebrates at the 2nd Interior Chamber of Praileaitz I site (Deba, Gipuzkoa).

Lechol 2

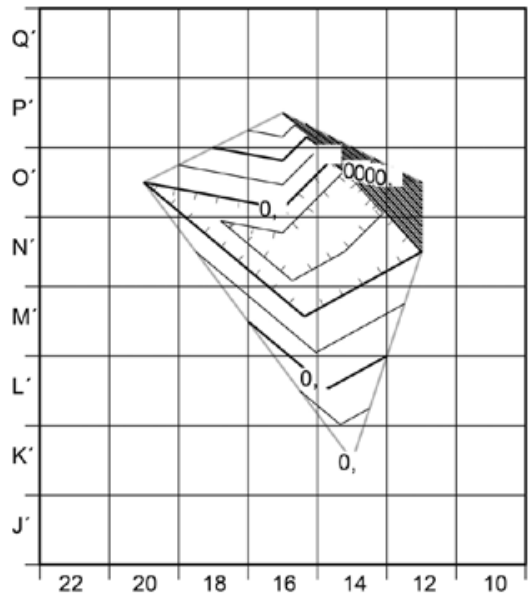

Lecho 7

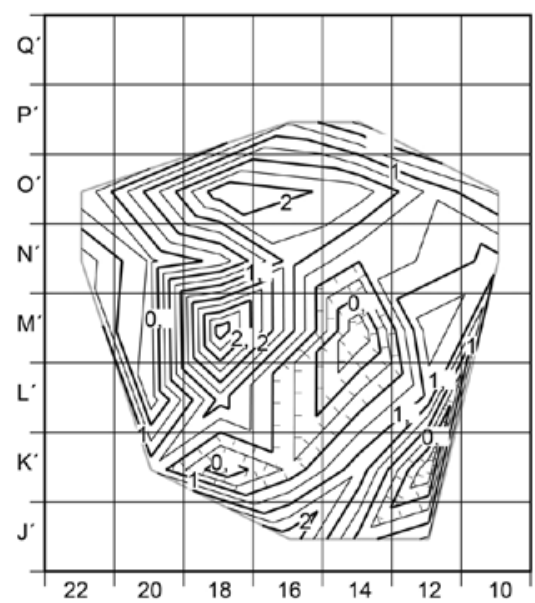

Lecho 5

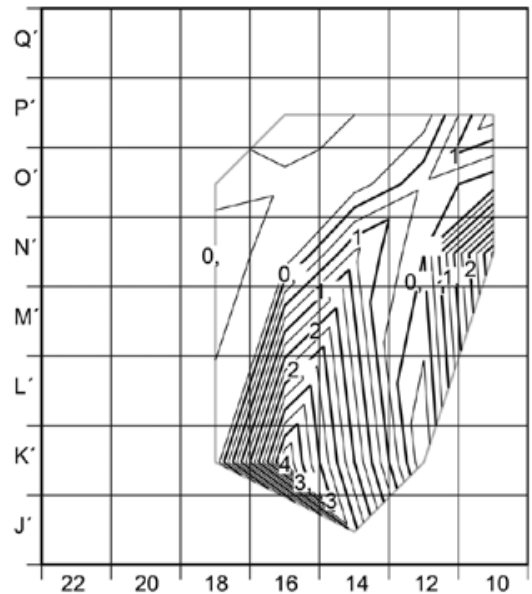

Lecho 8

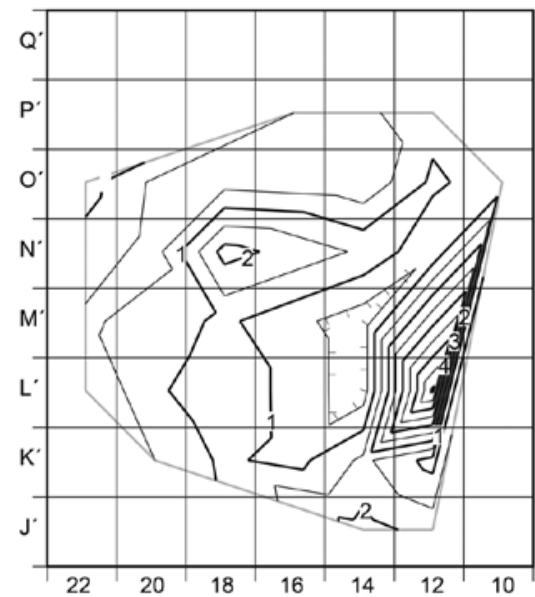

Lecho 6

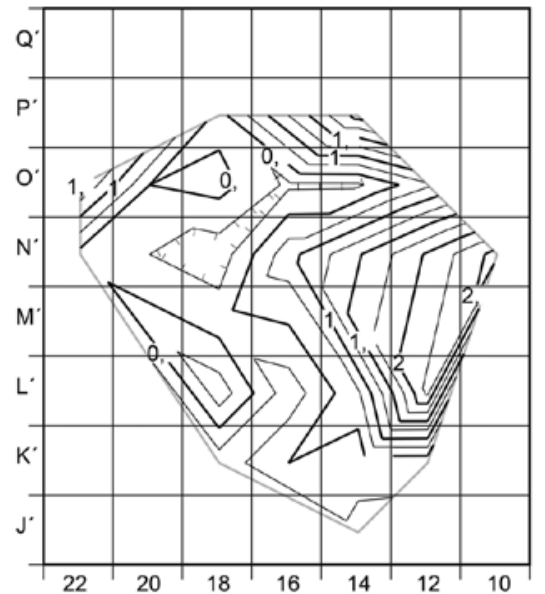

